\title{
Advantages of Two-Dimensional Electron-Induced Dissociation and Infrared Multiphoton Dissociation Mass Spectrometry for the Analysis of Agrochemicals - Supporting Information
}

\author{
Bryan P. Marzullo ${ }^{1}$, Tomos E. Morgan ${ }^{1}$, Christopher A. Wootton ${ }^{1}$, Simon Perry ${ }^{2}$, Mansoor Saeed ${ }^{2}$, \\ Mark P. Barrow ${ }^{1}$, Peter B. O'Connor ${ }^{1}$ \\ 1. Department of Chemistry, University of Warwick, Coventry, CV4 7AL, United Kingdom; \\ 2. Product Metabolism \& Analytical Sciences, Jealott's Hill International Research Centre, Syngenta, \\ Bracknell, Berkshire, RG42 6EY, United Kingdom
}

\section{Contents}

Table S 1: Table showing the peak list, relative intensity, assignments, and errors (ppm) of the IRMPD auto-correlation line. * displays the peaks that were used for the internal calibration.

Table S 2: Table showing the peak list, relative intensity, assignments, and errors (ppm) of the EID auto-correlation line. ${ }^{*}$ displays the peaks that were used for the internal calibration.

Table S 3. Table showing the peak list, relative intensity, assignments, and errors (ppm) of the IRMPD fragmentation of Azoxystrobin. * displays the peaks that were used for the internal calibration

Table S 4. Table showing the peak list, relative intensity, assignments, and errors (ppm) of the EID fragmentation of Azoxystrobin. ${ }^{*}$ displays the peaks that were used for the internal calibration....... 7 Table S 5. Table showing the peak list, relative intensity, assignments, and errors (ppm) of the IRMPD fragmentation of Fenpropridin. * displays the peaks that were used for the internal calibration.

Table S 6. Table showing the peak list, relative intensity, assignments, and errors (ppm) of the EID fragmentation of Fenpropridin. ${ }^{*}$ displays the peaks that were used for the internal calibration..... 10 Table S 7. Table showing the peak list, relative intensity, assignments, and errors (ppm) of the IRMPD fragmentation of Fluazifop acid. * displays the peaks that were used for the internal calibration

Table S 8. Table showing the peak list, relative intensity, assignments, and errors (ppm) of the EID fragmentation of Fluazifop acid. * displays the peaks that were used for the internal calibration.... 12 Table S 9. Table showing the peak list, relative intensity, assignments, and errors (ppm) of the IRMPD fragmentation of Fluazifop-p-butyl. * displays the peaks that were used for the internal calibration.

Table S 10. Table showing the peak list, relative intensity, assignments, and errors (ppm) of the EID fragmentation of Fluazifop-p-butyl. * displays the peaks that were used for the internal calibration.

Table S 11. Table showing the peak list, relative intensity, assignments, and errors (ppm) of the IRMPD fragmentation of Nicosulfuron. * displays the peaks that were used for the internal calibration.

Table S 12. Table showing the peak list, relative intensity, assignments, and errors (ppm) of the EID fragmentation of Nicosulfuron. ${ }^{*}$ displays the peaks that were used for the internal calibration. .... 16 Table S 13. Table showing the peak list, relative intensity, assignments, and errors (ppm) of the IRMPD fragmentation of Pirimiphos-methyl. * displays the peaks that were used for the internal calibration.

Table S 14. Table showing the peak list, relative intensity, assignments, and errors (ppm) of the EID fragmentation of Pirimiphos-methyl. ${ }^{*}$ displays the peaks that were used for the internal calibration. 
Table S 15. Table showing the peak list, relative intensity, assignments, and errors (ppm) of the IRMPD fragmentation of S-metolachlor. ${ }^{*}$ displays the peaks that were used for the internal calibration.

Table S 16. Table showing the peak list, relative intensity, assignments, and errors (ppm) of the EID fragmentation of S-metolachlor. ${ }^{*}$ displays the peaks that were used for the internal calibration. .. 20

Figure S 1. Extracted fragment line spectra for fenpropidin along with their cleavage assignment diagrams for a) IRMPD and b) EID.

Figure S 2. Extracted fragment line spectra for fluazifop acid along with their cleavage assignment diagrams for a) IRMPD and b) EID.

Figure S 3. Extracted fragment line spectra for fluazifop-p-butyl along with their cleavage assignment diagrams for a) IRMPD and b) EID.

Figure S 4. Extracted fragment line spectra for nicosulfuron along with their cleavage assignment diagrams for a) IRMPD and b) EID.

Figure S 5. Extracted fragment line spectra for S-metolachlor along with their cleavage assignment diagrams for a) IRMPD and b) EID.

Equation S 1: The equation used to calculate the fragmentation efficiency (FE) in percentage of the fragmentation method for each agrochemical. 
Table S 1: Table showing the peak list, relative intensity, assignments, and errors ( $\mathrm{ppm}$ ) of the IRMPD auto-correlation line. * displays the peaks that were used for the internal calibration.

\begin{tabular}{|c|c|c|c|c|c|c|}
\hline & $m / z$ & $\begin{array}{l}\text { Relative } \\
\text { Intensity }\end{array}$ & $\begin{array}{c}\text { Elemental } \\
\text { Composition }\end{array}$ & $\begin{array}{c}\text { Theoretical } \\
\text { Mass }\end{array}$ & $\begin{array}{l}\text { Error } \\
\text { (ppm) }\end{array}$ & Assignments \\
\hline & 433.08999 & 12.19 & $\mathrm{C} 15 \mathrm{H} 1806 \mathrm{~N} 6 \mathrm{~S}+\mathrm{Na}$ & 433.09007 & -0.194 & 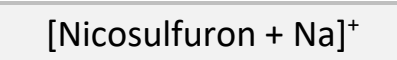 \\
\hline \multirow[t]{3}{*}{ * } & 411.10813 & 30.24 & $\mathrm{C} 15 \mathrm{H} 1806 \mathrm{~N} 6 \mathrm{~S}+\mathrm{H}$ & 411.10813 & 0.000 & {$[\text { Nicosulfuron }+\mathrm{H}]^{+}$} \\
\hline & 406.12368 & 7.29 & $\mathrm{C} 19 \mathrm{H} 2004 \mathrm{NF} 3+\mathrm{Na}$ & 406.12366 & 0.042 & 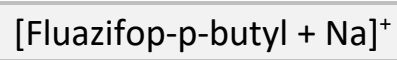 \\
\hline & 404.12408 & 27.92 & $\mathrm{C} 22 \mathrm{H} 17 \mathrm{~N} 3 \mathrm{O} 5+\mathrm{H}$ & 404.12410 & -0.042 & 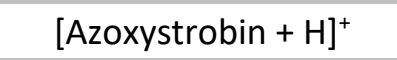 \\
\hline \multirow[t]{4}{*}{ * } & 384.14172 & 13.25 & $\mathrm{C} 19 \mathrm{H} 2004 \mathrm{NF} 3+\mathrm{H}$ & 384.14172 & 0.003 & 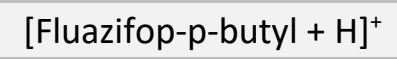 \\
\hline & 350.06079 & 1.99 & $\mathrm{C} 15 \mathrm{H} 12 \mathrm{O} 4 \mathrm{NF} 3+\mathrm{Na}$ & 350.06106 & -0.780 & 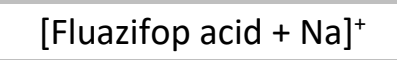 \\
\hline & 328.07801 & 4.69 & $\mathrm{C} 15 \mathrm{H} 12 \mathrm{O} 4 \mathrm{NF} 3+\mathrm{H}$ & 328.07912 & $-3.380^{a}$ & 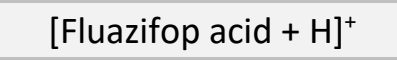 \\
\hline & 306.12320 & 3.62 & $\mathrm{C} 15 \mathrm{H} 22 \mathrm{CINO} 2+\mathrm{Na}$ & 306.12313 & 0.238 & 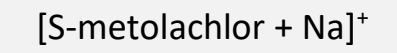 \\
\hline \multirow[t]{2}{*}{ * } & 306.10358 & 4.00 & $\mathrm{C} 11 \mathrm{H} 20 \mathrm{~N} 3 \mathrm{O} 3 \mathrm{PS}+\mathrm{H}$ & 306.10358 & 0.013 & {$[\text { Pirimiphos-methyl }+\mathrm{H}]^{+}$} \\
\hline & 284.14127 & 1.08 & $\mathrm{C} 15 \mathrm{H} 22 \mathrm{CINO} 2+\mathrm{H}$ & 284.14118 & 0.306 & 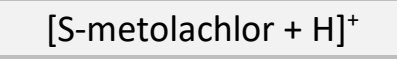 \\
\hline \multirow[t]{3}{*}{ * } & 274.25293 & 100.00 & $\mathrm{C} 19 \mathrm{H} 31 \mathrm{~N}+\mathrm{H}$ & 274.25293 & 0.015 & 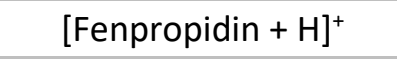 \\
\hline & & & & $\begin{array}{l}\text { Standard } \\
\text { Deviation }\end{array}$ & 1.045 & \\
\hline & & & & $\begin{array}{l}\text { Absolute } \\
\text { Average }\end{array}$ & 0.456 & \\
\hline
\end{tabular}

${ }^{a}$ This peak is composed of two overlapping species, protonated fluazifop acid and sodiated pirimiphos-methyl. These species are not resolved, which affects the assignment error. The sodiated pirimiphos-methyl does not display product ions, therefore the fragmentation line of the protonated fluazifop acid does not show product ions related to the pirimiphos-methyl molecule. 
Table S 2: Table showing the peak list, relative intensity, assignments, and errors (ppm) of the EID auto-correlation line. ${ }^{*}$ displays the peaks that were used for the internal calibration.

\begin{tabular}{|c|c|c|c|c|c|c|}
\hline & $m / z$ & $\begin{array}{l}\text { Relative } \\
\text { Intensity }\end{array}$ & $\begin{array}{c}\text { Elemental } \\
\text { Composition }\end{array}$ & $\begin{array}{l}\text { Theoretical } \\
\text { Mass }\end{array}$ & $\begin{array}{l}\text { Error } \\
(\mathrm{ppm})\end{array}$ & Assignments \\
\hline \multirow[t]{5}{*}{ * } & 433.09008 & 29.92 & $\mathrm{C} 15 \mathrm{H} 1806 \mathrm{~N} 6 \mathrm{~S}+\mathrm{Na}$ & 433.09007 & 0.014 & 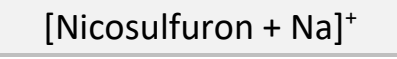 \\
\hline & 426.10594 & 11.59 & $\mathrm{C} 22 \mathrm{H} 17 \mathrm{~N} 3 \mathrm{O} 5+\mathrm{Na}$ & 426.10604 & -0.237 & \\
\hline & 411.10811 & 22.69 & $\mathrm{C} 15 \mathrm{H} 1806 \mathrm{~N} 6 \mathrm{~S}+\mathrm{H}$ & 411.10813 & -0.049 & 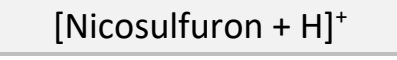 \\
\hline & 406.12373 & 6.07 & $\mathrm{C} 19 \mathrm{H} 2004 \mathrm{NF} 3+\mathrm{Na}$ & 406.12366 & 0.165 & {$[\text { Fluazifop-p-butyl }+\mathrm{Na}]^{+}$} \\
\hline & 404.12393 & 40.66 & $\mathrm{C} 22 \mathrm{H} 17 \mathrm{~N} 3 \mathrm{O} 5+\mathrm{H}$ & 404.12410 & -0.413 & 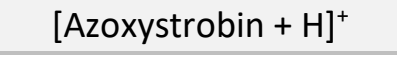 \\
\hline \multirow[t]{4}{*}{ * } & 384.14171 & 17.25 & $\mathrm{C} 19 \mathrm{H} 2004 \mathrm{NF} 3+\mathrm{H}$ & 384.14172 & -0.023 & 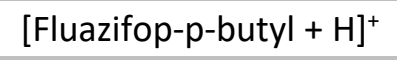 \\
\hline & 350.06120 & 14.32 & $\mathrm{C} 15 \mathrm{H} 12 \mathrm{O} 4 \mathrm{NF} 3+\mathrm{Na}$ & 350.06106 & 0.391 & 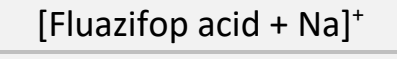 \\
\hline & 328.07796 & 48.09 & $\mathrm{C} 15 \mathrm{H} 12 \mathrm{O} 4 \mathrm{NF} 3+\mathrm{H}$ & 328.07912 & $-3.533^{a}$ & 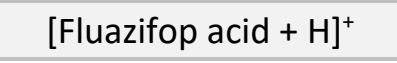 \\
\hline & 306.12321 & 6.28 & $\mathrm{C} 15 \mathrm{H} 22 \mathrm{CINO} 2+\mathrm{Na}$ & 306.12313 & 0.271 & 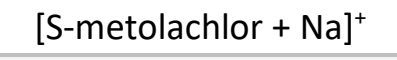 \\
\hline \multirow[t]{2}{*}{ * } & 306.10358 & 48.39 & $\mathrm{C} 11 \mathrm{H} 20 \mathrm{~N} 3 \mathrm{O} 3 \mathrm{PS}+\mathrm{H}$ & 306.10358 & 0.013 & {$[\text { Pirimiphos-methyl }+\mathrm{H}]^{+}$} \\
\hline & 284.14120 & 5.13 & $\mathrm{C} 15 \mathrm{H} 22 \mathrm{ClNO} 2+\mathrm{H}$ & 284.14118 & 0.060 & {$[\mathrm{~S}-\text { metolachlor }+\mathrm{H}]^{+}$} \\
\hline \multirow[t]{3}{*}{$*$} & 274.25293 & 100.00 & $\mathrm{C} 19 \mathrm{H} 31 \mathrm{~N}+\mathrm{H}$ & 274.25293 & 0.015 & 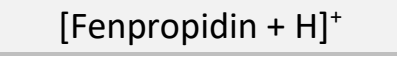 \\
\hline & & & & $\begin{array}{l}\text { Standard } \\
\text { Deviation }\end{array}$ & 1.047 & \\
\hline & & & & $\begin{array}{l}\text { Absolute } \\
\text { Average }\end{array}$ & 0.432 & \\
\hline
\end{tabular}

a This peak is composed of two overlapping species, protonated fluazifop acid and sodiated pirimiphos-methyl. These species are not resolved, which affects the assignment error. The sodiated pirimiphos-methyl does not display product ions, therefore the fragmentation line of the protonated fluazifop acid does not show product ions related to the pirimiphos-methyl molecule. 
Table S 3. Table showing the peak list, relative intensity, assignments, and errors (ppm) of the IRMPD fragmentation of Azoxystrobin.

\begin{tabular}{|c|c|c|c|c|c|}
\hline$m / z$ & $\begin{array}{l}\text { Relative } \\
\text { Intensity }\end{array}$ & $\begin{array}{l}\text { Elemental } \\
\text { Composition }\end{array}$ & $\begin{array}{c}\text { Theoretical } \\
\text { Mass }\end{array}$ & $\begin{array}{l}\text { Error } \\
\text { (ppm) }\end{array}$ & Assignments \\
\hline 404.12419 & 100.00 & $\mathrm{C}_{22} \mathrm{H}_{18} \mathrm{~N}_{3} \mathrm{O}_{5}$ & 404.12410 & 0.235 & {$[\mathrm{M}+\mathrm{H}]^{+}$} \\
\hline 372.09789 & 45.99 & $\mathrm{C}_{21} \mathrm{H}_{14} \mathrm{~N}_{3} \mathrm{O}_{4}$ & 372.09788 & 0.009 & A \\
\hline 344.10303 & 22.50 & $\mathrm{C}_{20} \mathrm{H}_{14} \mathrm{~N}_{3} \mathrm{O}_{3}$ & 344.10297 & 0.167 & B \\
\hline 340.07174 & 0.32 & $\mathrm{C}_{20} \mathrm{H}_{10} \mathrm{~N}_{3} \mathrm{O}_{3}$ & 340.07167 & 0.199 & AA \\
\hline 331.07141 & 0.41 & $\mathrm{C}_{19} \mathrm{H}_{11} \mathrm{~N}_{2} \mathrm{O}_{4}$ & 331.07133 & 0.225 & \\
\hline 329.07920 & 1.05 & $\mathrm{C}_{19} \mathrm{H}_{11} \mathrm{~N}_{3} \mathrm{O}_{3}$ & 329.07949 & -0.878 & \\
\hline 328.10808 & 1.94 & $\mathrm{C}_{20} \mathrm{H}_{14} \mathrm{~N}_{3} \mathrm{O}_{2}$ & 328.10805 & 0.088 & \\
\hline 328.07167 & 0.83 & $\mathrm{C}_{19} \mathrm{H}_{10} \mathrm{~N}_{3} \mathrm{O}_{3}$ & 328.07167 & -0.008 & $A C$ \\
\hline 326.09241 & 0.85 & $\mathrm{C}_{20} \mathrm{H}_{12} \mathrm{~N}_{3} \mathrm{O}_{2}$ & 326.09240 & 0.028 & \\
\hline 317.09208 & 6.79 & $\mathrm{C}_{19} \mathrm{H}_{13} \mathrm{~N}_{2} \mathrm{O}_{3}$ & 317.09207 & 0.028 & $\mathrm{BD}$ \\
\hline 316.10805 & 13.04 & $\mathrm{C}_{19} \mathrm{H}_{14} \mathrm{~N}_{3} \mathrm{O}_{2}$ & 316.10805 & -0.016 & $A B$ \\
\hline 315.10026 & 0.21 & $\mathrm{C}_{19} \mathrm{H}_{13} \mathrm{~N}_{3} \mathrm{O}_{2}$ & 315.10023 & 0.103 & \\
\hline 315.07644 & 1.39 & $\mathrm{C}_{19} \mathrm{H}_{11} \mathrm{~N}_{2} \mathrm{O}_{3}$ & 315.07642 & 0.065 & $\mathrm{BE}$ \\
\hline 314.09253 & 0.06 & $\mathrm{C}_{19} \mathrm{H}_{12} \mathrm{~N}_{3} \mathrm{O}_{2}$ & 314.09240 & 0.397 & $A B$ \\
\hline 313.06076 & 4.80 & $\mathrm{C}_{19} \mathrm{H}_{9} \mathrm{~N}_{2} \mathrm{O}_{3}$ & 313.06077 & -0.027 & $\mathrm{BE}$ \\
\hline 312.07673 & 1.61 & $\mathrm{C}_{19} \mathrm{H}_{10} \mathrm{~N}_{3} \mathrm{O}_{2}$ & 312.07675 & -0.068 & $A B$ \\
\hline 311.06892 & 0.80 & $\mathrm{C}_{19} \mathrm{H}_{9} \mathrm{~N}_{3} \mathrm{O}_{2}$ & 311.06893 & -0.024 & \\
\hline 303.07640 & 13.57 & $\mathrm{C}_{18} \mathrm{H}_{11} \mathrm{~N}_{2} \mathrm{O}_{3}$ & 303.07642 & -0.075 & $A C D$ \\
\hline 301.09713 & 1.36 & $\mathrm{C}_{19} \mathrm{H}_{13} \mathrm{~N}_{2} \mathrm{O}_{2}$ & 301.09715 & -0.082 & \\
\hline 301.08458 & 1.25 & $\mathrm{C}_{18} \mathrm{H}_{11} \mathrm{~N}_{3} \mathrm{O}_{2}$ & 301.08458 & -0.009 & \\
\hline 300.11315 & 0.17 & $\mathrm{C}_{19} \mathrm{H}_{14} \mathrm{~N}_{3} \mathrm{O}$ & 300.11314 & 0.041 & \\
\hline 299.08146 & 0.25 & $\mathrm{C}_{19} \mathrm{H}_{11} \mathrm{~N}_{2} \mathrm{O}_{2}$ & 299.08150 & -0.152 & \\
\hline 298.09746 & 0.11 & $\mathrm{C}_{19} \mathrm{H}_{12} \mathrm{~N}_{3} \mathrm{O}$ & 298.09749 & -0.088 & \\
\hline 289.09716 & 0.46 & $\mathrm{C}_{18} \mathrm{H}_{13} \mathrm{~N}_{2} \mathrm{O}_{2}$ & 289.09715 & 0.025 & $\mathrm{ABE} / \mathrm{ABD}$ \\
\hline 288.11316 & 0.41 & $\mathrm{C}_{18} \mathrm{H}_{14} \mathrm{~N}_{3} \mathrm{O}$ & 288.11314 & 0.083 & \\
\hline 288.06547 & 0.15 & $\mathrm{C}_{18} \mathrm{H}_{10} \mathrm{NO}_{3}$ & 288.06552 & -0.167 & $\mathrm{ACH}$ \\
\hline 287.08151 & 8.94 & $\mathrm{C}_{18} \mathrm{H}_{11} \mathrm{~N}_{2} \mathrm{O}_{2}$ & 287.08150 & 0.006 & $\mathrm{ABE} / \mathrm{ABD}$ \\
\hline 285.06581 & 0.13 & $\mathrm{C}_{18} \mathrm{H}_{9} \mathrm{~N}_{2} \mathrm{O}_{2}$ & 285.06585 & -0.170 & $\mathrm{ABE} / \mathrm{ABD}$ \\
\hline 275.08161 & 0.12 & $\mathrm{C}_{17} \mathrm{H}_{11} \mathrm{~N}_{2} \mathrm{O}_{2}$ & 275.08150 & 0.371 & $\mathrm{BCD}$ \\
\hline 274.08627 & 3.25 & $\mathrm{C}_{18} \mathrm{H}_{12} \mathrm{NO}_{2}$ & 274.08626 & 0.057 & $\mathrm{ABH}$ \\
\hline 273.08943 & 0.05 & $\mathrm{C}_{17} \mathrm{H}_{11} \mathrm{~N}_{3} \mathrm{O}$ & 273.08966 & -0.847 & \\
\hline 273.06582 & 0.13 & $\mathrm{C}_{17} \mathrm{H}_{9} \mathrm{~N}_{2} \mathrm{O}_{2}$ & 273.06585 & -0.125 & $\mathrm{BCD}$ \\
\hline 272.07064 & 0.10 & $\mathrm{C}_{18} \mathrm{H}_{10} \mathrm{NO}_{2}$ & 272.07061 & 0.136 & $\mathrm{ABH}$ \\
\hline 271.08656 & 0.18 & $\mathrm{C}_{18} \mathrm{H}_{11} \mathrm{~N}_{2} \mathrm{O}$ & 271.08659 & -0.123 & \\
\hline 260.07065 & 0.12 & $\mathrm{C}_{17} \mathrm{H}_{10} \mathrm{NO}_{2}$ & 260.07061 & 0.158 & $\mathrm{BCH}$ \\
\hline 259.08658 & 0.36 & $\mathrm{C}_{17} \mathrm{H}_{11} \mathrm{~N}_{2} \mathrm{O}$ & 259.08659 & -0.039 & \\
\hline 244.07557 & 0.07 & $\mathrm{C}_{17} \mathrm{H}_{10} \mathrm{NO}$ & 244.07569 & -0.493 & \\
\hline 239.04515 & 0.04 & $\mathrm{C}_{13} \mathrm{H}_{7} \mathrm{~N}_{2} \mathrm{O}_{3}$ & 239.04512 & 0.149 & AAl \\
\hline
\end{tabular}




\begin{tabular}{|c|c|c|c|c|c|}
\hline 223.07398 & 0.12 & $\mathrm{C}_{13} \mathrm{H}_{9} \mathrm{~N}_{3} \mathrm{O}$ & 223.07401 & -0.161 & \\
\hline 222.06616 & 0.09 & $\mathrm{C}_{13} \mathrm{H}_{8} \mathrm{~N}_{3} \mathrm{O}$ & 222.06619 & -0.106 & \\
\hline 211.07404 & 0.15 & $\mathrm{C}_{12} \mathrm{H}_{9} \mathrm{~N}_{3} \mathrm{O}$ & 211.07401 & 0.122 & \\
\hline 210.06616 & 0.38 & $\mathrm{C}_{12} \mathrm{H}_{8} \mathrm{~N}_{3} \mathrm{O}$ & 210.06619 & -0.149 & \\
\hline 210.04236 & 0.08 & $\mathrm{C}_{12} \mathrm{H}_{6} \mathrm{~N}_{2} \mathrm{O}_{2}$ & 210.04238 & -0.076 & ACM \\
\hline 202.04987 & 0.26 & $\mathrm{C}_{11} \mathrm{H}_{8} \mathrm{NO}_{3}$ & 202.04987 & 0.002 & $A G$ \\
\hline 201.06583 & 0.48 & $\mathrm{C}_{11} \mathrm{H}_{9} \mathrm{~N}_{2} \mathrm{O}_{2}$ & 201.06585 & -0.136 & $\mathrm{BF}$ \\
\hline 199.05016 & 0.15 & $\mathrm{C}_{11} \mathrm{H}_{7} \mathrm{~N}_{2} \mathrm{O}_{2}$ & 199.05020 & -0.209 & $\mathrm{BF}$ \\
\hline 197.07094 & 0.11 & $\mathrm{C}_{12} \mathrm{H}_{9} \mathrm{~N}_{2} \mathrm{O}$ & 197.07094 & -0.009 & $A B M$ \\
\hline 172.03930 & 2.00 & $\mathrm{C}_{10} \mathrm{H}_{6} \mathrm{NO}_{2}$ & 172.03930 & -0.040 & $\mathrm{HJ}^{\prime}$ \\
\hline 158.06004 & 0.04 & $\mathrm{C}_{10} \mathrm{H}_{8} \mathrm{NO}$ & 158.06004 & -0.001 & $\mathrm{HL}$ \\
\hline 145.02838 & 0.03 & $\mathrm{C}_{9} \mathrm{H}_{5} \mathrm{O}_{2}$ & 145.02841 & -0.174 & $\mathrm{DHJ} / \mathrm{HIQ}$ \\
\hline 134.06003 & 0.05 & $\mathrm{C}_{8} \mathrm{H}_{8} \mathrm{NO}$ & 134.06004 & -0.083 & \\
\hline & & & $\begin{array}{l}\text { Standard } \\
\text { Deviation }\end{array}$ & 0.225 & \\
\hline & & & $\begin{array}{l}\text { Absolute } \\
\text { Average }\end{array}$ & 0.140 & \\
\hline
\end{tabular}


Table S 4. Table showing the peak list, relative intensity, assignments, and errors (ppm) of the EID fragmentation of Azoxystrobin.

\begin{tabular}{|c|c|c|c|c|c|}
\hline$m / z$ & $\begin{array}{l}\text { Relative } \\
\text { Intensity }\end{array}$ & $\begin{array}{c}\text { Elemental } \\
\text { Composition }\end{array}$ & Theoretical Mass & $\begin{array}{l}\text { Error } \\
(\mathrm{ppm})\end{array}$ & Assignments \\
\hline 404.12151 & 100.00 & $\mathrm{C}_{22} \mathrm{H}_{18} \mathrm{~N}_{3} \mathrm{O}_{5}$ & 404.12410 & $-6.406^{a}$ & {$[\mathrm{M}+\mathrm{H}]+$} \\
\hline 372.09659 & 4.19 & $\mathrm{C}_{21} \mathrm{H}_{14} \mathrm{~N}_{3} \mathrm{O}_{4}$ & 372.09788 & $-3.468^{a}$ & A \\
\hline 357.07442 & 0.28 & $\mathrm{C}_{20} \mathrm{H}_{11} \mathrm{~N}_{3} \mathrm{O}_{4}$ & 357.07441 & 0.022 & $A R$ \\
\hline 344.10283 & 1.74 & $\mathrm{C}_{20} \mathrm{H}_{14} \mathrm{~N}_{3} \mathrm{O}_{3}$ & 344.10297 & -0.411 & B \\
\hline 340.07162 & 0.64 & $\mathrm{C}_{20} \mathrm{H}_{10} \mathrm{~N}_{3} \mathrm{O}_{3}$ & 340.07167 & -0.133 & AA \\
\hline 328.10819 & 0.10 & $\mathrm{C}_{20} \mathrm{H}_{14} \mathrm{~N}_{3} \mathrm{O}_{2}$ & 328.10805 & 0.427 & \\
\hline 328.07156 & 0.92 & $\mathrm{C}_{19} \mathrm{H}_{10} \mathrm{~N}_{3} \mathrm{O}_{3}$ & 328.07167 & -0.342 & $A C$ \\
\hline 326.09259 & 0.07 & $\mathrm{C}_{20} \mathrm{H}_{12} \mathrm{~N}_{3} \mathrm{O}_{2}$ & 326.09240 & 0.575 & \\
\hline 317.09213 & 0.37 & $\mathrm{C}_{19} \mathrm{H}_{13} \mathrm{~N}_{2} \mathrm{O}_{3}$ & 317.09207 & 0.187 & $\mathrm{BD}$ \\
\hline 316.10801 & 0.94 & $\mathrm{C}_{19} \mathrm{H}_{14} \mathrm{~N}_{3} \mathrm{O}_{2}$ & 316.10805 & -0.152 & $A B$ \\
\hline 315.07617 & 0.09 & $\mathrm{C}_{19} \mathrm{H}_{11} \mathrm{~N}_{2} \mathrm{O}_{3}$ & 315.07642 & -0.801 & $\mathrm{BE}$ \\
\hline 313.06076 & 0.23 & $\mathrm{C}_{19} \mathrm{H}_{9} \mathrm{~N}_{2} \mathrm{O}_{3}$ & 313.06077 & -0.018 & $\mathrm{BE}$ \\
\hline 312.07671 & 0.42 & $\mathrm{C}_{19} \mathrm{H}_{10} \mathrm{~N}_{3} \mathrm{O}_{2}$ & 312.07675 & -0.142 & $A B$ \\
\hline 311.06883 & 0.04 & $\mathrm{C}_{19} \mathrm{H}_{9} \mathrm{~N}_{3} \mathrm{O}_{2}$ & 311.06893 & -0.303 & \\
\hline 303.07636 & 0.76 & $\mathrm{C}_{18} \mathrm{H}_{11} \mathrm{~N}_{2} \mathrm{O}_{3}$ & 303.07642 & -0.192 & $A C D$ \\
\hline 301.09720 & 0.10 & $\mathrm{C}_{19} \mathrm{H}_{13} \mathrm{~N}_{2} \mathrm{O}_{2}$ & 301.09715 & 0.166 & \\
\hline 301.08451 & 0.58 & $\mathrm{C}_{18} \mathrm{H}_{11} \mathrm{~N}_{3} \mathrm{O}_{2}$ & 301.08458 & -0.215 & \\
\hline 300.07672 & 0.55 & $\mathrm{C}_{18} \mathrm{H}_{10} \mathrm{~N}_{3} \mathrm{O}_{2}$ & 300.07675 & -0.113 & \\
\hline 289.09712 & 0.05 & $\mathrm{C}_{18} \mathrm{H}_{13} \mathrm{~N}_{2} \mathrm{O}_{2}$ & 289.09715 & -0.113 & $\mathrm{ABE} / \mathrm{ABD}$ \\
\hline 287.08146 & 0.65 & $\mathrm{C}_{18} \mathrm{H}_{11} \mathrm{~N}_{2} \mathrm{O}_{2}$ & 287.08150 & -0.161 & $\mathrm{ABE} / \mathrm{ABD}$ \\
\hline 284.08160 & 0.08 & $\mathrm{C}_{18} \mathrm{H}_{10} \mathrm{~N}_{3} \mathrm{O}$ & 284.08184 & -0.850 & \\
\hline 275.08156 & 0.08 & $\mathrm{C}_{17} \mathrm{H}_{11} \mathrm{~N}_{2} \mathrm{O}_{2}$ & 275.08150 & 0.219 & $\mathrm{BCD}$ \\
\hline 274.08633 & 0.10 & $\mathrm{C}_{18} \mathrm{H}_{12} \mathrm{NO}_{2}$ & 274.08626 & 0.258 & $\mathrm{ABH}$ \\
\hline 273.08953 & 0.17 & $\mathrm{C}_{17} \mathrm{H}_{11} \mathrm{~N}_{3} \mathrm{O}$ & 273.08966 & -0.471 & \\
\hline 273.06590 & 0.37 & $\mathrm{C}_{17} \mathrm{H}_{9} \mathrm{~N}_{2} \mathrm{O}_{2}$ & 273.06585 & 0.176 & $B C D$ \\
\hline 272.08182 & 0.32 & $\mathrm{C}_{17} \mathrm{H}_{10} \mathrm{~N}_{3} \mathrm{O}$ & 272.08184 & -0.062 & \\
\hline 271.08652 & 0.05 & $\mathrm{C}_{18} \mathrm{H}_{11} \mathrm{~N}_{2} \mathrm{O}$ & 271.08659 & -0.239 & \\
\hline 259.08648 & 0.05 & $\mathrm{C}_{17} \mathrm{H}_{11} \mathrm{~N}_{2} \mathrm{O}$ & 259.08659 & -0.418 & \\
\hline 258.07894 & 0.07 & $\mathrm{C}_{17} \mathrm{H}_{10} \mathrm{~N}_{2} \mathrm{O}$ & 258.07876 & 0.662 & \\
\hline 257.07110 & 0.12 & $\mathrm{C}_{17} \mathrm{H}_{9} \mathrm{~N}_{2} \mathrm{O}$ & 257.07094 & 0.626 & \\
\hline 254.06836 & 0.06 & $\mathrm{C}_{14} \mathrm{H}_{10} \mathrm{~N}_{2} \mathrm{O}_{3}$ & 254.06859 & -0.935 & \\
\hline 245.07103 & 0.12 & $\mathrm{C}_{16} \mathrm{H}_{9} \mathrm{~N}_{2} \mathrm{O}$ & 245.07094 & 0.389 & \\
\hline 239.04511 & 0.07 & $\mathrm{C}_{13} \mathrm{H}_{7} \mathrm{~N}_{2} \mathrm{O}_{3}$ & 239.04512 & -0.017 & AAl \\
\hline 229.07593 & 0.10 & $\mathrm{C}_{16} \mathrm{H}_{9} \mathrm{~N}_{2}$ & 229.07602 & -0.399 & \\
\hline 224.04557 & 0.06 & $\mathrm{C}_{12} \mathrm{H}_{6} \mathrm{~N}_{3} \mathrm{O}_{2}$ & 224.04545 & 0.520 & \\
\hline 216.06548 & 0.42 & $\mathrm{C}_{12} \mathrm{H}_{10} \mathrm{NO}_{3}$ & 216.06552 & -0.174 & \\
\hline 212.03424 & 0.39 & $\mathrm{C}_{12} \mathrm{H}_{6} \mathrm{NO}_{3}$ & 212.03422 & 0.108 & \\
\hline 211.05020 & 0.07 & $\mathrm{C}_{12} \mathrm{H}_{7} \mathrm{~N}_{2} \mathrm{O}_{2}$ & 211.05020 & -0.011 & DP \\
\hline 210.06618 & 0.06 & $\mathrm{C}_{12} \mathrm{H}_{8} \mathrm{~N}_{3} \mathrm{O}$ & 210.06619 & -0.054 & \\
\hline 210.04238 & 0.61 & $\mathrm{C}_{12} \mathrm{H}_{6} \mathrm{~N}_{2} \mathrm{O}_{2}$ & 210.04238 & 0.017 & ACM \\
\hline
\end{tabular}




\begin{tabular}{|c|c|c|c|c|c|}
\hline$m / z$ & $\begin{array}{l}\text { Relative } \\
\text { Intensity }\end{array}$ & $\begin{array}{c}\text { Elemental } \\
\text { Composition }\end{array}$ & Theoretical Mass & $\begin{array}{l}\text { Error } \\
\text { (ppm) }\end{array}$ & Assignments \\
\hline 201.06587 & 0.10 & $\mathrm{C}_{11} \mathrm{H}_{9} \mathrm{~N}_{2} \mathrm{O}_{2}$ & 201.06585 & 0.102 & $\mathrm{BF}$ \\
\hline 201.04210 & 0.21 & $\mathrm{C}_{11} \mathrm{H}_{7} \mathrm{NO}_{3}$ & 201.04204 & 0.261 & \\
\hline 200.03420 & 0.12 & $\mathrm{C}_{11} \mathrm{H}_{6} \mathrm{NO}_{3}$ & 200.03422 & -0.121 & \\
\hline 199.05013 & 0.13 & $\mathrm{C}_{11} \mathrm{H}_{7} \mathrm{~N}_{2} \mathrm{O}_{2}$ & 199.05020 & -0.390 & $\mathrm{BF}$ \\
\hline 197.07095 & 0.06 & $\mathrm{C}_{12} \mathrm{H}_{9} \mathrm{~N}_{2} \mathrm{O}$ & 197.07094 & 0.032 & ABM \\
\hline 196.05054 & 0.12 & $\mathrm{C}_{11} \mathrm{H}_{6} \mathrm{~N}_{3} \mathrm{O}$ & 196.05054 & 0.017 & $\mathrm{~L}$ \\
\hline 191.07029 & 0.67 & $\mathrm{C}_{11} \mathrm{H}_{11} \mathrm{O}_{3}$ & 191.07027 & 0.104 & $\mathrm{~J}$ \\
\hline 183.05532 & 0.15 & $\mathrm{C}_{11} \mathrm{H}_{7} \mathrm{~N}_{2} \mathrm{O}$ & 183.05529 & 0.144 & \\
\hline 176.04679 & 0.22 & $\mathrm{C}_{10} \mathrm{H}_{8} \mathrm{O}_{3}$ & 176.04680 & -0.033 & \\
\hline 172.03934 & 0.73 & $\mathrm{C}_{10} \mathrm{H}_{6} \mathrm{NO}_{2}$ & 172.03930 & 0.188 & $H J^{\prime}$ \\
\hline 171.05534 & 0.07 & $\mathrm{C}_{10} \mathrm{H}_{7} \mathrm{~N}_{2} \mathrm{O}$ & 171.05529 & 0.320 & $\mathrm{DL} / \mathrm{MQ}$ \\
\hline 169.03964 & 0.03 & $\mathrm{C}_{10} \mathrm{H}_{5} \mathrm{~N}_{2} \mathrm{O}$ & 169.03964 & -0.003 & $\mathrm{DL} / \mathrm{MQ}$ \\
\hline 156.04434 & 0.17 & $\mathrm{C}_{10} \mathrm{H}_{6} \mathrm{NO}$ & 156.04439 & -0.311 & $\mathrm{HL}$ \\
\hline 147.04406 & 0.14 & $\mathrm{C}_{9} \mathrm{H}_{7} \mathrm{O}_{2}$ & 147.04406 & 0.054 & \\
\hline 145.02841 & 0.16 & $\mathrm{C}_{9} \mathrm{H}_{5} \mathrm{O}_{2}$ & 145.02841 & -0.003 & $\mathrm{DHJ} / \mathrm{HIQ}$ \\
\hline & & & Standard Deviation & 0.574 & \\
\hline & & & Absolute Average & 0.308 & \\
\hline
\end{tabular}

${ }^{a}$ The error values of these assignments are high due to the high intensities of these peaks. 
Table S 5. Table showing the peak list, relative intensity, assignments, and errors (ppm) of the IRMPD fragmentation of Fenpropridin.

\begin{tabular}{|c|c|c|c|c|c|}
\hline$m / z$ & $\begin{array}{l}\text { Relative } \\
\text { Intensity }\end{array}$ & $\begin{array}{l}\text { Elemental } \\
\text { Composition }\end{array}$ & $\begin{array}{c}\text { Theoretical } \\
\text { Mass }\end{array}$ & $\begin{array}{l}\text { Error } \\
\text { (ppm) }\end{array}$ & Assignments \\
\hline 274.25323 & 100.00 & $\mathrm{C} 19 \mathrm{H} 32 \mathrm{~N}$ & 274.25293 & $1.114^{a}$ & {$[\mathrm{M}+\mathrm{H}]+$} \\
\hline 258.22162 & 0.02 & $\mathrm{C} 18 \mathrm{H} 28 \mathrm{~N}$ & 258.22163 & -0.008 & A \\
\hline 218.19047 & 2.12 & $\mathrm{C} 15 \mathrm{H} 24 \mathrm{~N}$ & 218.19033 & 0.650 & B \\
\hline 216.17479 & 0.05 & $\mathrm{C} 15 \mathrm{H} 22 \mathrm{~N}$ & 216.17468 & 0.507 & B \\
\hline 189.16383 & 0.17 & $\mathrm{C} 14 \mathrm{H} 21$ & 189.16378 & 0.300 & C \\
\hline 161.13248 & 0.16 & $\mathrm{C} 12 \mathrm{H} 17$ & 161.13248 & 0.027 & $A D$ \\
\hline 147.11682 & 3.14 & $\mathrm{C} 11 \mathrm{H} 15$ & 147.11683 & -0.053 & $E$ \\
\hline 133.10119 & 0.06 & $\mathrm{C} 10 \mathrm{H} 13$ & 133.10118 & 0.130 & \\
\hline 132.09335 & 0.02 & $\mathrm{C} 10 \mathrm{H} 12$ & 132.09335 & -0.034 & $F$ \\
\hline 131.08559 & 0.01 & $\mathrm{C} 10 \mathrm{H} 11$ & 131.08553 & 0.506 & $B C$ \\
\hline 119.08547 & 0.02 & $\mathrm{C} 9 \mathrm{H} 11$ & 119.08553 & -0.455 & $\mathrm{AF} / \mathrm{BD}$ \\
\hline \multirow[t]{3}{*}{117.06988} & 0.04 & $\mathrm{C9H} 9$ & 117.06988 & 0.014 & $A F / B D$ \\
\hline & & & $\begin{array}{l}\text { Standard } \\
\text { Deviation }\end{array}$ & 0.320 & \\
\hline & & & $\begin{array}{l}\text { Absolute } \\
\text { Average }\end{array}$ & 0.244 & \\
\hline
\end{tabular}

${ }^{a}$ The error value of this assignment is high due to the high intensity of this peak. 
Table S 6. Table showing the peak list, relative intensity, assignments, and errors (ppm) of the EID fragmentation of Fenpropridin.

\begin{tabular}{|c|c|c|c|c|c|}
\hline$m / z$ & $\begin{array}{l}\text { Relative } \\
\text { Intensity }\end{array}$ & $\begin{array}{c}\text { Elemental } \\
\text { Composition }\end{array}$ & Theoretical Mass & $\begin{array}{l}\text { Error } \\
\text { (ppm) }\end{array}$ & Assignments \\
\hline 274.25210 & 100.00 & $\mathrm{C}_{19} \mathrm{H}_{32} \mathrm{~N}$ & 274.25293 & -3.022 & {$[\mathrm{M}+\mathrm{H}]+$} \\
\hline 272.23742 & 0.18 & $\mathrm{C}_{19} \mathrm{H}_{30} \mathrm{~N}$ & 272.23728 & 0.527 & \\
\hline 260.25029 & 0.16 & $\mathrm{C}_{19} \mathrm{H}_{32}$ & 260.24985 & 1.675 & \\
\hline 258.22163 & 0.23 & $\mathrm{C}_{18} \mathrm{H}_{28} \mathrm{~N}$ & 258.22163 & 0.010 & A \\
\hline 254.20320 & 0.03 & $\mathrm{C}_{19} \mathrm{H}_{26}$ & 254.20290 & 1.170 & \\
\hline 246.23455 & 0.18 & $\mathrm{C}_{18} \mathrm{H}_{30}$ & 246.23420 & 1.411 & \\
\hline 232.21892 & 0.13 & $\mathrm{C}_{17} \mathrm{H}_{28}$ & 232.21855 & 1.592 & \\
\hline 218.19032 & 0.12 & $\mathrm{C}_{15} \mathrm{H}_{24} \mathrm{~N}$ & 218.19033 & -0.008 & B \\
\hline 217.18237 & 0.03 & $\mathrm{C}_{15} \mathrm{H}_{23} \mathrm{~N}$ & 217.18250 & -0.595 & \\
\hline 216.17464 & 0.01 & $\mathrm{C}_{15} \mathrm{H}_{22} \mathrm{~N}$ & 216.17468 & -0.184 & B \\
\hline 211.14853 & 0.02 & $\mathrm{C}_{16} \mathrm{H}_{19}$ & 211.14813 & 1.926 & \\
\hline 203.17950 & 0.05 & $\mathrm{C}_{15} \mathrm{H}_{23}$ & 203.17943 & 0.345 & \\
\hline 197.13271 & 0.06 & $\mathrm{C}_{15} \mathrm{H}_{17}$ & 197.13248 & 1.207 & \\
\hline 189.16385 & 0.05 & $\mathrm{C}_{14} \mathrm{H}_{21}$ & 189.16378 & 0.401 & C \\
\hline 175.14827 & 0.04 & $\mathrm{C}_{13} \mathrm{H}_{19}$ & 175.14813 & 0.803 & D \\
\hline 173.13266 & 0.03 & $\mathrm{C}_{13} \mathrm{H}_{17}$ & 173.13248 & 1.051 & D \\
\hline 161.13257 & 0.05 & $\mathrm{C}_{12} \mathrm{H}_{17}$ & 161.13248 & 0.604 & $A D / A A C$ \\
\hline 159.11700 & 0.05 & $\mathrm{C}_{12} \mathrm{H}_{15}$ & 159.11683 & 1.112 & $A D / A A C$ \\
\hline 147.11683 & 0.33 & $\mathrm{C}_{11} \mathrm{H}_{15}$ & 147.11683 & -0.012 & $\mathrm{AAD} / \mathrm{E}$ \\
\hline 145.10117 & 0.06 & $\mathrm{C}_{11} \mathrm{H}_{13}$ & 145.10118 & -0.054 & $\mathrm{AAD} / \mathrm{E}$ \\
\hline 133.10126 & 0.04 & $\mathrm{C}_{10} \mathrm{H}_{13}$ & 133.10118 & 0.638 & \\
\hline 132.09329 & 0.07 & $\mathrm{C}_{10} \mathrm{H}_{12}$ & 132.09335 & -0.495 & $\mathrm{~F}$ \\
\hline 131.08547 & 0.06 & $\mathrm{C}_{10} \mathrm{H}_{11}$ & 131.08553 & -0.438 & $\mathrm{BC}$ \\
\hline 126.12766 & 0.06 & $\mathrm{C}_{8} \mathrm{H}_{16} \mathrm{~N}$ & 126.12773 & -0.522 & $\mathrm{E}^{\prime}$ \\
\hline 119.08553 & 0.09 & $\mathrm{C}_{9} \mathrm{H}_{11}$ & 119.08553 & 0.018 & $\mathrm{AF} / \mathrm{BD}$ \\
\hline 117.06987 & 0.11 & $\mathrm{C}_{9} \mathrm{H}_{9}$ & 117.06988 & -0.091 & AF/BD \\
\hline 115.05417 & 0.03 & $\mathrm{C}_{9} \mathrm{H}_{7}$ & 115.05423 & -0.499 & AF/BD \\
\hline 105.06988 & 0.04 & $\mathrm{C}_{8} \mathrm{H}_{9}$ & 105.06988 & -0.008 & AAF \\
\hline \multirow{2}{*}{ 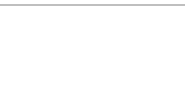 } & & & Standard Deviation & 0.749 & \\
\hline & & & Absolute Average & 0.644 & \\
\hline
\end{tabular}


Table S 7. Table showing the peak list, relative intensity, assignments, and errors (ppm) of the IRMPD fragmentation of Fluazifop acid.

\begin{tabular}{|c|c|c|c|c|c|}
\hline$m / z$ & $\begin{array}{l}\text { Relative } \\
\text { Intensity }\end{array}$ & $\begin{array}{c}\text { Elemental } \\
\text { Composition }\end{array}$ & Theoretical Mass & $\begin{array}{l}\text { Error } \\
\text { (ppm) }\end{array}$ & Assignments \\
\hline 328.07792 & 100.00 & $\mathrm{C}_{15} \mathrm{H}_{13} \mathrm{~F}_{3} \mathrm{NO}_{4}$ & 328.07912 & $-3.667^{a}$ & {$[\mathrm{M}+\mathrm{H}]+$} \\
\hline 282.07364 & 65.46 & $\mathrm{C}_{14} \mathrm{H}_{11} \mathrm{~F}_{3} \mathrm{NO}_{2}$ & 282.07364 & 0.013 & B \\
\hline 268.05799 & 1.42 & $\mathrm{C}_{13} \mathrm{H}_{9} \mathrm{~F}_{3} \mathrm{NO}_{2}$ & 268.05799 & -0.016 & $\mathrm{BC}$ \\
\hline 264.06303 & 0.70 & $\mathrm{C}_{14} \mathrm{H}_{9} \mathrm{~F}_{3} \mathrm{NO}$ & 264.06308 & -0.173 & Rearrangement \\
\hline 256.05791 & 1.31 & $\mathrm{C}_{12} \mathrm{H}_{9} \mathrm{~F}_{3} \mathrm{NO}_{2}$ & 256.05799 & -0.320 & D \\
\hline 255.05012 & 0.77 & $\mathrm{C}_{12} \mathrm{H}_{8} \mathrm{~F}_{3} \mathrm{NO}_{2}$ & 255.05016 & -0.182 & \\
\hline 254.07858 & 0.84 & $\mathrm{C}_{13} \mathrm{H}_{11} \mathrm{~F}_{3} \mathrm{NO}$ & 254.07873 & -0.576 & Rearrangement \\
\hline 254.04232 & 5.91 & $\mathrm{C}_{12} \mathrm{H}_{7} \mathrm{~F}_{3} \mathrm{NO}_{2}$ & 254.04234 & -0.095 & D \\
\hline 238.04729 & 1.38 & $\mathrm{C}_{12} \mathrm{H}_{7} \mathrm{~F}_{3} \mathrm{NO}$ & 238.04742 & -0.554 & $E$ \\
\hline 227.05516 & 1.27 & $\mathrm{C}_{11} \mathrm{H}_{8} \mathrm{~F}_{3} \mathrm{NO}$ & 227.05525 & -0.390 & $\mathrm{H}$ \\
\hline 226.04734 & 0.36 & $\mathrm{C}_{11} \mathrm{H}_{7} \mathrm{~F}_{3} \mathrm{NO}$ & 226.04742 & -0.390 & \\
\hline 218.04120 & 0.21 & $\mathrm{C}_{12} \mathrm{H}_{6} \mathrm{~F}_{2} \mathrm{NO}$ & 218.04120 & 0.003 & EM \\
\hline 213.07839 & 0.96 & $\mathrm{C}_{13} \mathrm{H}_{11} \mathrm{NO}_{2}$ & 213.07843 & -0.174 & $\mathrm{BL}$ \\
\hline 198.05254 & 0.20 & $\mathrm{C}_{10} \mathrm{H}_{7} \mathrm{~F}_{3} \mathrm{~N}$ & 198.05251 & 0.155 & Rearrangement \\
\hline 147.02904 & 0.65 & $\mathrm{C}_{6} \mathrm{H}_{4} \mathrm{~F}_{3} \mathrm{~N}$ & 147.02904 & 0.000 & $\mathrm{G}$ \\
\hline 121.02845 & 0.61 & $\mathrm{C}_{7} \mathrm{H}_{5} \mathrm{O}_{2}$ & 121.02841 & 0.361 & $\mathrm{BCG}^{\prime}$ \\
\hline \multirow{2}{*}{ 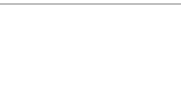 } & & & Standard Deviation & 0.259 & \\
\hline & & & Absolute Average & 0.227 & \\
\hline
\end{tabular}

${ }^{a}$ The error value of this assignment is high due to the high intensity of this peak. 
Table S 8. Table showing the peak list, relative intensity, assignments, and errors (ppm) of the EID fragmentation of Fluazifop acid.

\begin{tabular}{|c|c|c|c|c|c|}
\hline$m / z$ & Relative Intensity & $\begin{array}{l}\text { Elemental } \\
\text { Composition }\end{array}$ & Theoretical Mass & Error (ppm) & Assignments \\
\hline 328.07825 & 100.00 & $\mathrm{C}_{15} \mathrm{H}_{13} \mathrm{~F}_{3} \mathrm{NO}_{4}$ & 328.07912 & $-2.656^{a}$ & {$[\mathrm{M}+\mathrm{H}]+$} \\
\hline 282.07364 & 2.56 & $\mathrm{C}_{14} \mathrm{H}_{11} \mathrm{~F}_{3} \mathrm{NO}_{2}$ & 282.07364 & -0.003 & B \\
\hline 268.05810 & 1.61 & $\mathrm{C}_{13} \mathrm{H}_{9} \mathrm{~F}_{3} \mathrm{NO}_{2}$ & 268.05799 & 0.408 & $\mathrm{BC}$ \\
\hline 256.05813 & 0.48 & $\mathrm{C}_{12} \mathrm{H}_{9} \mathrm{~F}_{3} \mathrm{NO}_{2}$ & 256.05799 & 0.552 & $\mathrm{D}$ \\
\hline 255.05026 & 0.99 & $\mathrm{C}_{12} \mathrm{H}_{8} \mathrm{~F}_{3} \mathrm{NO}_{2}$ & 255.05016 & 0.367 & \\
\hline 254.07879 & 0.32 & $\mathrm{C}_{13} \mathrm{H}_{11} \mathrm{~F}_{3} \mathrm{NO}$ & 254.07873 & 0.239 & Rearrangement \\
\hline 254.04246 & 1.94 & $\mathrm{C}_{12} \mathrm{H}_{7} \mathrm{~F}_{3} \mathrm{NO}_{2}$ & 254.04234 & 0.489 & D \\
\hline 240.06308 & 0.31 & $\mathrm{C}_{12} \mathrm{H}_{9} \mathrm{~F}_{3} \mathrm{NO}$ & 240.06308 & 0.004 & $\mathrm{E}$ \\
\hline 239.05511 & 0.12 & $\mathrm{C}_{12} \mathrm{H}_{8} \mathrm{~F}_{3} \mathrm{NO}$ & 239.05525 & -0.584 & \\
\hline 238.04745 & 1.01 & $\mathrm{C}_{12} \mathrm{H}_{7} \mathrm{~F}_{3} \mathrm{NO}$ & 238.04742 & 0.112 & $E$ \\
\hline 236.06803 & 0.07 & $\mathrm{C}_{13} \mathrm{H}_{9} \mathrm{~F}_{3} \mathrm{~N}$ & 236.06816 & -0.537 & Rearrangement \\
\hline 227.05529 & 0.60 & $\mathrm{C}_{11} \mathrm{H}_{8} \mathrm{~F}_{3} \mathrm{NO}$ & 227.05525 & 0.171 & $\mathrm{H}$ \\
\hline 226.04748 & 0.48 & $\mathrm{C}_{11} \mathrm{H}_{7} \mathrm{~F}_{3} \mathrm{NO}$ & 226.04742 & 0.231 & \\
\hline 218.04123 & 0.20 & $\mathrm{C}_{12} \mathrm{H}_{6} \mathrm{~F}_{2} \mathrm{NO}$ & 218.04120 & 0.140 & EM \\
\hline 214.04754 & 0.03 & $\mathrm{C}_{10} \mathrm{H}_{7} \mathrm{~F}_{3} \mathrm{NO}$ & 214.04742 & 0.552 & $\mathrm{~K}$ \\
\hline 213.07839 & 0.16 & $\mathrm{C}_{13} \mathrm{H}_{11} \mathrm{NO}_{2}$ & 213.07843 & -0.205 & $\mathrm{BL}$ \\
\hline 211.06020 & 0.04 & $\mathrm{C}_{11} \mathrm{H}_{8} \mathrm{~F}_{3} \mathrm{~N}$ & 211.06034 & -0.642 & \\
\hline 210.05267 & 0.06 & $\mathrm{C}_{11} \mathrm{H}_{7} \mathrm{~F}_{3} \mathrm{~N}$ & 210.05251 & 0.746 & Rearrangement \\
\hline 200.03157 & 0.03 & $\mathrm{C}_{9} \mathrm{H}_{5} \mathrm{~F}_{3} \mathrm{NO}$ & 200.03177 & -1.042 & $\mathrm{~N}$ \\
\hline 199.06039 & 0.03 & $\mathrm{C}_{10} \mathrm{H}_{8} \mathrm{~F}_{3} \mathrm{~N}$ & 199.06034 & 0.267 & Rearrangement \\
\hline 198.05251 & 0.32 & $\mathrm{C}_{10} \mathrm{H}_{7} \mathrm{~F}_{3} \mathrm{~N}$ & 198.05251 & 0.011 & Rearrangement \\
\hline 190.04646 & 0.06 & $\mathrm{C}_{11} \mathrm{H}_{6} \mathrm{~F}_{2} \mathrm{~N}$ & 190.04628 & 0.927 & Rearrangement \\
\hline 186.05237 & 0.05 & $\mathrm{C}_{9} \mathrm{H}_{7} \mathrm{~F}_{3} \mathrm{~N}$ & 186.05251 & -0.744 & Rearrangement \\
\hline 182.05736 & 0.50 & $\mathrm{C}_{9} \mathrm{H}_{10} \mathrm{O}_{4}$ & 182.05736 & -0.001 & $\mathrm{G}^{\prime}$ \\
\hline 178.04628 & 0.05 & $\mathrm{C}_{10} \mathrm{H}_{6} \mathrm{~F}_{2} \mathrm{~N}$ & 178.04628 & -0.010 & \\
\hline 164.03173 & 0.20 & $\mathrm{C}_{6} \mathrm{H}_{5} \mathrm{~F}_{3} \mathrm{NO}$ & 164.03177 & -0.265 & $\mathrm{~F}$ \\
\hline 163.02391 & 0.03 & $\mathrm{C}_{6} \mathrm{H}_{4} \mathrm{~F}_{3} \mathrm{NO}$ & 163.02395 & -0.266 & \\
\hline 147.02900 & 0.48 & $\mathrm{C}_{6} \mathrm{H}_{4} \mathrm{~F}_{3} \mathrm{~N}$ & 147.02904 & -0.250 & G \\
\hline 146.02121 & 0.20 & $\mathrm{C}_{6} \mathrm{H}_{3} \mathrm{~F}_{3} \mathrm{~N}$ & 146.02121 & -0.032 & \\
\hline 127.02280 & 0.03 & $\mathrm{C}_{6} \mathrm{H}_{3} \mathrm{~F}_{2} \mathrm{~N}$ & 127.02281 & -0.023 & GM \\
\hline 110.03623 & 0.05 & $\mathrm{C}_{6} \mathrm{H}_{6} \mathrm{O}_{2}$ & 110.03623 & 0.000 & $\mathrm{DG}^{\prime}$ \\
\hline \multirow[t]{2}{*}{ 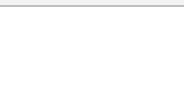 } & & & Standard Deviation & 0.444 & \\
\hline & & & Absolute Average & 0.327 & \\
\hline
\end{tabular}

${ }^{a}$ The error value of this assignment is high due to the high intensity of this peak. 
Table S 9. Table showing the peak list, relative intensity, assignments, and errors (ppm) of the IRMPD fragmentation of Fluazifop-p-butyl.

\begin{tabular}{|c|c|c|c|c|c|}
\hline$m / z$ & $\begin{array}{l}\text { Relative } \\
\text { Intensity }\end{array}$ & $\begin{array}{c}\text { Elemental } \\
\text { Composition }\end{array}$ & Theoretical Mass & $\begin{array}{l}\text { Error } \\
\text { (ppm) }\end{array}$ & Assignments \\
\hline 384.14192 & 100.00 & $\mathrm{C}_{19} \mathrm{H}_{20} \mathrm{~F}_{3} \mathrm{NO}_{4} \mathrm{H}$ & 384.14172 & 0.510 & {$[\mathrm{M}+\mathrm{H}]+$} \\
\hline 338.13647 & 0.22 & $\mathrm{C}_{18} \mathrm{H}_{19} \mathrm{~F}_{3} \mathrm{NO}_{2}$ & 338.13624 & 0.695 & \\
\hline 328.07912 & 13.29 & $\mathrm{C}_{15} \mathrm{H}_{13} \mathrm{~F}_{3} \mathrm{NO}_{4}$ & 328.07912 & -0.004 & $A$ \\
\hline 310.06858 & 0.62 & $\mathrm{C}_{15} \mathrm{H}_{11} \mathrm{~F}_{3} \mathrm{NO}_{3}$ & 310.06855 & 0.099 & $\mathrm{~J}$ \\
\hline 282.07354 & 7.82 & $\mathrm{C}_{14} \mathrm{H}_{11} \mathrm{~F}_{3} \mathrm{NO}_{2}$ & 282.07364 & -0.354 & B \\
\hline 264.06313 & 1.26 & $\mathrm{C}_{14} \mathrm{H}_{9} \mathrm{~F}_{3} \mathrm{NO}$ & 264.06308 & 0.191 & Rearrangement \\
\hline 256.05808 & 0.17 & $\mathrm{C}_{12} \mathrm{H}_{9} \mathrm{~F}_{3} \mathrm{NO}_{2}$ & 256.05799 & 0.355 & D \\
\hline 254.07878 & 3.85 & $\mathrm{C}_{13} \mathrm{H}_{11} \mathrm{~F}_{3} \mathrm{NO}$ & 254.07873 & 0.208 & Rearrangement \\
\hline 254.04247 & 0.17 & $\mathrm{C}_{12} \mathrm{H}_{7} \mathrm{~F}_{3} \mathrm{NO}_{2}$ & 254.04234 & 0.498 & D \\
\hline 240.06312 & 0.41 & $\mathrm{C}_{12} \mathrm{H}_{9} \mathrm{~F}_{3} \mathrm{NO}$ & 240.06308 & 0.174 & $\mathrm{E}$ \\
\hline 239.05525 & 0.22 & $\mathrm{C}_{12} \mathrm{H}_{8} \mathrm{~F}_{3} \mathrm{NO}$ & 239.05525 & 0.002 & \\
\hline 238.04743 & 6.32 & $\mathrm{C}_{12} \mathrm{H}_{7} \mathrm{~F}_{3} \mathrm{NO}$ & 238.04742 & 0.016 & $E$ \\
\hline 236.06820 & 0.38 & $\mathrm{C}_{13} \mathrm{H}_{9} \mathrm{~F}_{3} \mathrm{~N}$ & 236.06816 & 0.153 & Rearrangement \\
\hline 214.04742 & 0.32 & $\mathrm{C}_{10} \mathrm{H}_{7} \mathrm{~F}_{3} \mathrm{NO}$ & 214.04742 & -0.013 & $\mathrm{~K}$ \\
\hline 213.07843 & 1.56 & $\mathrm{C}_{13} \mathrm{H}_{11} \mathrm{NO}_{2}$ & 213.07843 & -0.018 & $\mathrm{BL}$ \\
\hline 212.03184 & 0.23 & $\mathrm{C}_{10} \mathrm{H}_{5} \mathrm{~F}_{3} \mathrm{NO}$ & 212.03177 & 0.327 & $\mathrm{~K}$ \\
\hline 186.05250 & 0.27 & $\mathrm{C}_{9} \mathrm{H}_{7} \mathrm{~F}_{3} \mathrm{~N}$ & 186.05251 & -0.040 & Rearrangement \\
\hline 164.03178 & 0.16 & $\mathrm{C}_{6} \mathrm{H}_{5} \mathrm{~F}_{3} \mathrm{NO}$ & 164.03177 & 0.001 & $\mathrm{LF}$ \\
\hline 121.02846 & 0.56 & $\mathrm{C}_{7} \mathrm{H}_{5} \mathrm{O}_{2}$ & 121.02841 & 0.420 & $\mathrm{BCG}^{\prime}$ \\
\hline & & & Standard Deviation & 0.243 & \\
\hline & & & Absolute Average & 0.198 & \\
\hline
\end{tabular}


Table S 10. Table showing the peak list, relative intensity, assignments, and errors ( $\mathrm{ppm}$ ) of the EID fragmentation of Fluazifop-p-butyl.

\begin{tabular}{|c|c|c|c|c|c|}
\hline$m / z$ & $\begin{array}{l}\text { Relative } \\
\text { Intensity }\end{array}$ & $\begin{array}{l}\text { Elemental } \\
\text { Composition }\end{array}$ & Theoretical Mass & $\begin{array}{l}\text { Error } \\
(\mathrm{ppm})\end{array}$ & Assignments \\
\hline 384.14003 & 100.00 & $\mathrm{C}_{19} \mathrm{H}_{20} \mathrm{~F}_{3} \mathrm{NO}_{4} \mathrm{H}$ & 384.14172 & $-4.385^{a}$ & {$[\mathrm{M}+\mathrm{H}]+$} \\
\hline 383.13388 & 0.22 & $\mathrm{C}_{19} \mathrm{H}_{20} \mathrm{~F}_{3} \mathrm{NO}_{4}$ & 383.13389 & -0.048 & \\
\hline 282.07365 & 5.17 & $\mathrm{C}_{14} \mathrm{H}_{11} \mathrm{~F}_{3} \mathrm{NO}_{2}$ & 282.07364 & 0.024 & B \\
\hline 268.05795 & 3.38 & $\mathrm{C}_{13} \mathrm{H}_{9} \mathrm{~F}_{3} \mathrm{NO}_{2}$ & 268.05799 & -0.155 & $B C$ \\
\hline 256.05798 & 0.25 & $\mathrm{C}_{12} \mathrm{H}_{9} \mathrm{~F}_{3} \mathrm{NO}_{2}$ & 256.05799 & -0.038 & $\mathrm{D}$ \\
\hline 255.05006 & 0.63 & $\mathrm{C}_{12} \mathrm{H}_{8} \mathrm{~F}_{3} \mathrm{NO}_{2}$ & 255.05016 & -0.415 & \\
\hline 254.07869 & 0.55 & $\mathrm{C}_{13} \mathrm{H}_{11} \mathrm{~F}_{3} \mathrm{NO}$ & 254.07873 & -0.120 & Rearrangement \\
\hline 254.04230 & 0.91 & $\mathrm{C}_{12} \mathrm{H}_{7} \mathrm{~F}_{3} \mathrm{NO}_{2}$ & 254.04234 & -0.145 & D \\
\hline 240.06309 & 0.48 & $\mathrm{C}_{12} \mathrm{H}_{9} \mathrm{~F}_{3} \mathrm{NO}$ & 240.06308 & 0.076 & $E$ \\
\hline 238.12001 & 0.52 & $\mathrm{C}_{13} \mathrm{H}_{18} \mathrm{O}_{4}$ & 238.11996 & 0.212 & $\mathrm{G}^{\prime}$ \\
\hline 238.04740 & 0.68 & $\mathrm{C}_{12} \mathrm{H}_{7} \mathrm{~F}_{3} \mathrm{NO}$ & 238.04742 & -0.098 & $E$ \\
\hline 227.05520 & 0.32 & $\mathrm{C}_{11} \mathrm{H}_{8} \mathrm{~F}_{3} \mathrm{NO}$ & 227.05525 & -0.232 & \\
\hline 226.04748 & 0.11 & $\mathrm{C}_{11} \mathrm{H}_{7} \mathrm{~F}_{3} \mathrm{NO}$ & 226.04742 & 0.242 & $\mathrm{H}$ \\
\hline 218.04120 & 0.18 & $\mathrm{C}_{12} \mathrm{H}_{6} \mathrm{~F}_{2} \mathrm{NO}$ & 218.04120 & 0.015 & EM \\
\hline 147.02904 & 0.10 & $\mathrm{C}_{6} \mathrm{H}_{4} \mathrm{~F}_{3} \mathrm{~N}$ & 147.02904 & -0.001 & $G$ \\
\hline & & & Standard Deviation & 0.170 & \\
\hline & & & Absolute Average & 0.130 & \\
\hline
\end{tabular}

${ }^{a}$ The error value of this assignment is high due to the high intensity of this peak. 
Table S 11. Table showing the peak list, relative intensity, assignments, and errors (ppm) of the IRMPD fragmentation of Nicosulfuron.

\begin{tabular}{|c|c|c|c|c|c|}
\hline$m / z$ & $\begin{array}{l}\text { Relative } \\
\text { Intensity }\end{array}$ & $\begin{array}{l}\text { Elemental } \\
\text { Composition }\end{array}$ & Theoretical Mass & $\begin{array}{l}\text { Error } \\
\text { (ppm) }\end{array}$ & Assignments \\
\hline 411.10804 & 100.00 & $\mathrm{C}_{15} \mathrm{H}_{19} \mathrm{~N}_{6} \mathrm{O}_{6} \mathrm{~S}$ & 411.10813 & -0.223 & {$[\mathrm{M}+\mathrm{H}]+$} \\
\hline 366.05028 & 1.10 & $\mathrm{C}_{13} \mathrm{H}_{12} \mathrm{~N}_{5} \mathrm{O}_{6} \mathrm{~S}$ & 366.05028 & 0.000 & A \\
\hline 304.14022 & 0.18 & $\mathrm{C}_{14} \mathrm{H}_{18} \mathrm{~N}_{5} \mathrm{O}_{3}$ & 304.14042 & -0.630 & \\
\hline 259.08257 & 2.77 & $\mathrm{C}_{12} \mathrm{H}_{11} \mathrm{~N}_{4} \mathrm{O}_{3}$ & 259.08257 & 0.001 & Rearrangement \\
\hline 230.05939 & 0.17 & $\mathrm{C}_{8} \mathrm{H}_{12} \mathrm{~N}_{3} \mathrm{O}_{3} \mathrm{~S}$ & 230.05939 & -0.001 & $\mathrm{C}$ \\
\hline 227.11378 & 0.06 & $\mathrm{C}_{9} \mathrm{H}_{15} \mathrm{~N}_{4} \mathrm{O}_{3}$ & 227.11387 & -0.396 & \\
\hline 213.03283 & 12.04 & $\mathrm{C}_{8} \mathrm{H}_{9} \mathrm{~N}_{2} \mathrm{O}_{3} \mathrm{~S}$ & 213.03284 & -0.048 & $\mathrm{D}$ \\
\hline 182.05602 & 18.00 & $\mathrm{C}_{7} \mathrm{H}_{8} \mathrm{~N}_{3} \mathrm{O}_{3}$ & 182.05602 & 0.000 & $C^{\prime}$ \\
\hline & & & Standard Deviation & 0.255 & \\
\hline & & & Absolute Average & 0.154 & \\
\hline
\end{tabular}


Table S 12. Table showing the peak list, relative intensity, assignments, and errors ( $\mathrm{ppm}$ ) of the EID fragmentation of Nicosulfuron.

\begin{tabular}{|c|c|c|c|c|c|}
\hline$m / z$ & $\begin{array}{l}\text { Relative } \\
\text { Intensity }\end{array}$ & $\begin{array}{c}\text { Elemental } \\
\text { Composition }\end{array}$ & Theoretical Mass & $\begin{array}{l}\text { Error } \\
\text { (ppm) }\end{array}$ & Assignments \\
\hline 411.10884 & 100.00 & $\mathrm{C}_{15} \mathrm{H}_{19} \mathrm{~N}_{6} \mathrm{O}_{6} \mathrm{~S}$ & 411.10813 & 1.737 & {$[\mathrm{M}+\mathrm{H}]+$} \\
\hline 366.05028 & 0.41 & $\mathrm{C}_{13} \mathrm{H}_{12} \mathrm{~N}_{5} \mathrm{O}_{6} \mathrm{~S}$ & 366.05028 & 0.006 & A \\
\hline 213.03283 & 3.49 & $\mathrm{C}_{8} \mathrm{H}_{9} \mathrm{~N}_{2} \mathrm{O}_{3} \mathrm{~S}$ & 213.03284 & -0.034 & $\mathrm{D}$ \\
\hline 198.07475 & 0.36 & $\mathrm{C}_{7} \mathrm{H}_{10} \mathrm{~N}_{4} \mathrm{O}_{3}$ & 198.07474 & 0.029 & $D^{\prime}$ \\
\hline 182.05589 & 3.93 & $\mathrm{C}_{7} \mathrm{H}_{8} \mathrm{~N}_{3} \mathrm{O}_{3}$ & 182.05602 & -0.674 & $C^{\prime}$ \\
\hline 156.07662 & 0.87 & $\mathrm{C}_{6} \mathrm{H}_{10} \mathrm{~N}_{3} \mathrm{O}_{2}$ & 156.07675 & -0.864 & $B^{\prime}$ \\
\hline 155.06883 & 0.61 & $\mathrm{C}_{6} \mathrm{H}_{9} \mathrm{~N}_{3} \mathrm{O}_{2}$ & 155.06893 & -0.650 & \\
\hline 154.06100 & 0.87 & $\mathrm{C}_{6} \mathrm{H}_{8} \mathrm{~N}_{3} \mathrm{O}_{2}$ & 154.06110 & -0.671 & $\mathrm{~B}^{\prime}$ \\
\hline 139.04999 & 0.11 & $\mathrm{C}_{6} \mathrm{H}_{7} \mathrm{~N}_{2} \mathrm{O}_{2}$ & 139.05020 & -1.567 & $\mathrm{~F}$ \\
\hline \multirow[t]{3}{*}{106.02874} & 0.09 & $\mathrm{C}_{6} \mathrm{H}_{4} \mathrm{NO}$ & 106.02874 & 0.000 & $\mathrm{AE}$ \\
\hline & & & Standard Deviation & 0.543 & \\
\hline & & & Absolute Average & 0.499 & \\
\hline
\end{tabular}


Table S 13. Table showing the peak list, relative intensity, assignments, and errors (ppm) of the IRMPD fragmentation of Pirimiphos-methyl. * displays the peaks that were used for the internal calibration.

\begin{tabular}{|c|c|c|c|c|c|c|}
\hline & $m / z$ & $\begin{array}{l}\text { Relative } \\
\text { Intensity }\end{array}$ & $\begin{array}{c}\text { Elemental } \\
\text { Composition }\end{array}$ & Theoretical Mass & $\begin{array}{l}\text { Error } \\
\text { (ppm) }\end{array}$ & Assignments \\
\hline & 306.10336 & 100.00 & $\mathrm{C}_{11} \mathrm{H}_{21} \mathrm{~N}_{3} \mathrm{O}_{3} \mathrm{PS}$ & 306.10358 & -0.705 & {$[\mathrm{M}+\mathrm{H}]+$} \\
\hline$*$ & 278.07228 & 6.79 & $\mathrm{C}_{9} \mathrm{H}_{16} \mathrm{~N}_{3} \mathrm{O}_{3} \mathrm{PSH}$ & 278.07228 & 0.012 & A \\
\hline \multirow[t]{5}{*}{ * } & 274.07736 & 12.39 & $\mathrm{C}_{10} \mathrm{H}_{17} \mathrm{~N}_{3} \mathrm{O}_{2} \mathrm{PS}$ & 274.07736 & -0.003 & B \\
\hline & 262.04111 & 0.25 & $\mathrm{C}_{8} \mathrm{H}_{13} \mathrm{~N}_{3} \mathrm{O}_{3} \mathrm{PS}$ & 262.04098 & 0.519 & $\mathrm{AE}$ \\
\hline & 250.04094 & 0.38 & $\mathrm{C}_{7} \mathrm{H}_{13} \mathrm{~N}_{3} \mathrm{O}_{3} \mathrm{PS}$ & 250.04098 & -0.156 & $A A$ \\
\hline & 246.04605 & 4.01 & $\mathrm{C}_{8} \mathrm{H}_{13} \mathrm{~N}_{3} \mathrm{O}_{2} \mathrm{PS}$ & 246.04606 & -0.033 & $A B$ \\
\hline & 233.01442 & 0.70 & $\mathrm{C}_{7} \mathrm{H}_{10} \mathrm{~N}_{2} \mathrm{O}_{3} \mathrm{PS}$ & 233.01443 & -0.045 & $C$ \\
\hline \multirow[t]{6}{*}{$*$} & 218.01476 & 0.74 & $\mathrm{C}_{6} \mathrm{H}_{9} \mathrm{~N}_{3} \mathrm{O}_{2} \mathrm{PS}$ & 218.01476 & -0.016 & $A A B$ \\
\hline & 216.13831 & 2.00 & $\mathrm{C}_{10} \mathrm{H}_{21} \mathrm{~N}_{2} \mathrm{OP}$ & 216.13860 & -1.356 & \\
\hline & 214.11052 & 0.17 & $\mathrm{C}_{9} \mathrm{H}_{17} \mathrm{~N}_{3} \mathrm{OP}$ & 214.11038 & 0.679 & BBD \\
\hline & 197.09810 & 0.17 & $\mathrm{C}_{9} \mathrm{H}_{15} \mathrm{~N}_{3} \mathrm{~S}$ & 197.09812 & -0.113 & Rearrangement \\
\hline & 196.14450 & 0.49 & $\mathrm{C}_{10} \mathrm{H}_{18} \mathrm{~N}_{3} \mathrm{O}$ & 196.14444 & 0.298 & \\
\hline & 180.11311 & 0.12 & $\mathrm{C}_{9} \mathrm{H}_{14} \mathrm{~N}_{3} \mathrm{O}$ & 180.11314 & -0.163 & G \\
\hline \multirow[t]{3}{*}{$*$} & 164.11823 & 2.83 & $\mathrm{C}_{9} \mathrm{H}_{14} \mathrm{~N}_{3}$ & 164.11822 & 0.009 & $\mathrm{H}$ \\
\hline & 152.08180 & 0.33 & $\mathrm{C}_{7} \mathrm{H}_{10} \mathrm{~N}_{3} \mathrm{O}$ & 152.08184 & -0.226 & AG \\
\hline & 147.11668 & 0.38 & $\mathrm{C}_{11} \mathrm{H}_{15}$ & 147.11683 & -0.986 & \\
\hline \multirow[t]{3}{*}{$*$} & 124.98206 & 0.81 & $\mathrm{C}_{2} \mathrm{H}_{6} \mathrm{O}_{2} \mathrm{PS}$ & 124.98206 & -0.002 & $\mathrm{G}^{\prime}$ \\
\hline & & & & Standard Deviation & 0.489 & \\
\hline & & & & Absolute Average & 0.289 & \\
\hline
\end{tabular}


Table S 14. Table showing the peak list, relative intensity, assignments, and errors (ppm) of the EID fragmentation of Pirimiphos-methyl. * displays the peaks that were used for the internal calibration.

\begin{tabular}{|c|c|c|c|c|c|c|}
\hline & $m / z$ & $\begin{array}{l}\text { Relative } \\
\text { Intensity }\end{array}$ & $\begin{array}{l}\text { Elemental } \\
\text { Composition }\end{array}$ & Theoretical Mass & $\begin{array}{l}\text { Error } \\
\text { (ppm) }\end{array}$ & Assignments \\
\hline & 306.10242 & 100.00 & $\mathrm{C}_{11} \mathrm{H}_{21} \mathrm{~N}_{3} \mathrm{O}_{3} \mathrm{PS}$ & 306.10358 & -3.766 & {$[\mathrm{M}+\mathrm{H}]+$} \\
\hline & 290.07239 & 0.47 & $\mathrm{C}_{10} \mathrm{H}_{17} \mathrm{~N}_{3} \mathrm{O}_{3} \mathrm{PS}$ & 290.07228 & 0.411 & $\mathrm{E}$ \\
\hline \multirow[t]{10}{*}{$*$} & 278.07227 & 0.57 & $\mathrm{C}_{9} \mathrm{H}_{17} \mathrm{~N}_{3} \mathrm{O}_{3} \mathrm{PS}$ & 278.07228 & -0.009 & A \\
\hline & 276.05665 & 0.39 & $\mathrm{C}_{9} \mathrm{H}_{15} \mathrm{~N}_{3} \mathrm{O}_{3} \mathrm{PS}$ & 276.05663 & 0.075 & A \\
\hline & 274.13167 & 0.41 & $\mathrm{C}_{11} \mathrm{H}_{21} \mathrm{~N}_{3} \mathrm{O}_{3} \mathrm{P}$ & 274.13150 & 0.596 & D \\
\hline & 274.07733 & 0.60 & $\mathrm{C}_{10} \mathrm{H}_{17} \mathrm{~N}_{3} \mathrm{O}_{2} \mathrm{PS}$ & 274.07736 & -0.128 & B \\
\hline & 262.05342 & 0.09 & $\mathrm{C}_{9} \mathrm{H}_{15} \mathrm{~N}_{2} \mathrm{O}_{3} \mathrm{PS}$ & 262.05355 & -0.493 & $M$ \\
\hline & 262.04083 & 0.27 & $\mathrm{C}_{8} \mathrm{H}_{13} \mathrm{~N}_{3} \mathrm{O}_{3} \mathrm{PS}$ & 262.04098 & -0.556 & $\mathrm{AE}$ \\
\hline & 250.04110 & 0.15 & $\mathrm{C}_{7} \mathrm{H}_{13} \mathrm{~N}_{3} \mathrm{O}_{3} \mathrm{PS}$ & 250.04098 & 0.489 & AA \\
\hline & 246.04608 & 0.45 & $\mathrm{C}_{8} \mathrm{H}_{13} \mathrm{~N}_{3} \mathrm{O}_{2} \mathrm{PS}$ & 246.04606 & 0.059 & $A B$ \\
\hline & 244.03039 & 0.37 & $\mathrm{C}_{8} \mathrm{H}_{11} \mathrm{~N}_{3} \mathrm{O}_{2} \mathrm{PS}$ & 244.03041 & -0.088 & $A B$ \\
\hline & 242.10537 & 0.22 & $\mathrm{C}_{10} \mathrm{H}_{17} \mathrm{~N}_{3} \mathrm{O}_{2} \mathrm{P}$ & 242.10529 & 0.325 & BD \\
\hline \multirow[t]{15}{*}{$*$} & 233.01443 & 0.59 & $\mathrm{C}_{7} \mathrm{H}_{10} \mathrm{~N}_{2} \mathrm{O}_{3} \mathrm{PS}$ & 233.01443 & 0.012 & $C$ \\
\hline & 230.01457 & 0.11 & $\mathrm{C}_{7} \mathrm{H}_{9} \mathrm{~N}_{3} \mathrm{O}_{2} \mathrm{PS}$ & 230.01476 & -0.825 & ABE \\
\hline & 228.08968 & 0.12 & $\mathrm{C}_{9} \mathrm{H}_{15} \mathrm{~N}_{3} \mathrm{O}_{2} \mathrm{P}$ & 228.08964 & 0.165 & BDE \\
\hline & 218.01495 & 0.06 & $\mathrm{C}_{6} \mathrm{H}_{9} \mathrm{~N}_{3} \mathrm{O}_{2} \mathrm{PS}$ & 218.01476 & 0.872 & $A A B$ \\
\hline & 212.00441 & 0.06 & $\mathrm{C}_{7} \mathrm{H}_{7} \mathrm{~N}_{3} \mathrm{OPS}$ & 212.00420 & 0.991 & $A B B$ \\
\hline & 207.08940 & 0.14 & $\mathrm{C}_{7} \mathrm{H}_{16} \mathrm{~N}_{2} \mathrm{O}_{3} \mathrm{P}$ & 207.08931 & 0.465 & DEF \\
\hline & 197.09803 & 0.08 & $\mathrm{C}_{9} \mathrm{H}_{15} \mathrm{~N}_{3} \mathrm{~S}$ & 197.09812 & -0.459 & Rearrangement \\
\hline & 196.14446 & 0.16 & $\mathrm{C}_{10} \mathrm{H}_{18} \mathrm{~N}_{3} \mathrm{O}$ & 196.14444 & 0.124 & \\
\hline & 196.09034 & 0.14 & $\mathrm{C}_{9} \mathrm{H}_{14} \mathrm{~N}_{3} \mathrm{~S}$ & 196.09029 & 0.226 & Rearrangement \\
\hline & 195.13652 & 0.12 & $\mathrm{C}_{10} \mathrm{H}_{17} \mathrm{~N}_{3} \mathrm{O}$ & 195.13661 & -0.491 & \\
\hline & 182.12885 & 0.16 & $\mathrm{C}_{9} \mathrm{H}_{16} \mathrm{~N}_{3} \mathrm{O}$ & 182.12879 & 0.332 & G \\
\hline & 182.07458 & 0.08 & $\mathrm{C}_{8} \mathrm{H}_{12} \mathrm{~N}_{3} \mathrm{~S}$ & 182.07464 & -0.349 & Rearrangement \\
\hline & 181.12101 & 0.35 & $\mathrm{C}_{9} \mathrm{H}_{15} \mathrm{~N}_{3} \mathrm{O}$ & 181.12096 & 0.255 & \\
\hline & 180.11315 & 0.46 & $\mathrm{C}_{9} \mathrm{H}_{14} \mathrm{~N}_{3} \mathrm{O}$ & 180.11314 & 0.070 & G \\
\hline & 179.05809 & 0.03 & $\mathrm{C}_{5} \mathrm{H}_{12} \mathrm{~N}_{2} \mathrm{O}_{3} \mathrm{P}$ & 179.05801 & 0.488 & ADEF \\
\hline \multirow[t]{13}{*}{ * } & 168.11314 & 0.11 & $\mathrm{C}_{8} \mathrm{H}_{14} \mathrm{~N}_{3} \mathrm{O}$ & 168.11314 & -0.004 & EG \\
\hline & 168.05902 & 0.11 & $\mathrm{C}_{7} \mathrm{H}_{10} \mathrm{~N}_{3} \mathrm{~S}$ & 168.05899 & 0.155 & Rearrangement \\
\hline & 166.09747 & 0.07 & $\mathrm{C}_{8} \mathrm{H}_{12} \mathrm{~N}_{3} \mathrm{O}$ & 166.09749 & -0.098 & EG \\
\hline & 166.04342 & 0.02 & $\mathrm{C}_{7} \mathrm{H}_{8} \mathrm{~N}_{3} \mathrm{~S}$ & 166.04334 & 0.480 & Rearrangement \\
\hline & 165.12605 & 0.07 & $\mathrm{C}_{9} \mathrm{H}_{15} \mathrm{~N}_{3}$ & 165.12605 & 0.033 & \\
\hline & 164.11825 & 0.62 & $\mathrm{C}_{9} \mathrm{H}_{14} \mathrm{~N}_{3}$ & 164.11822 & 0.172 & $\mathrm{H}$ \\
\hline & 163.11050 & 0.06 & $\mathrm{C}_{9} \mathrm{H}_{13} \mathrm{~N}_{3}$ & 163.11040 & 0.643 & \\
\hline & 152.08186 & 0.28 & $\mathrm{C}_{7} \mathrm{H}_{10} \mathrm{~N}_{3} \mathrm{O}$ & 152.08184 & 0.170 & AG \\
\hline & 151.02672 & 0.13 & $\mathrm{C}_{3} \mathrm{H}_{8} \mathrm{~N}_{2} \mathrm{O}_{3} \mathrm{P}$ & 151.02671 & 0.097 & AADEF \\
\hline & 150.10263 & 0.18 & $\mathrm{C}_{8} \mathrm{H}_{12} \mathrm{~N}_{3}$ & 150.10257 & 0.368 & $\mathrm{EH}$ \\
\hline & 138.06627 & 0.05 & $\mathrm{C}_{6} \mathrm{H}_{8} \mathrm{~N}_{3} \mathrm{O}$ & 138.06619 & 0.617 & AEG \\
\hline & 136.08691 & 0.06 & $\mathrm{C}_{7} \mathrm{H}_{10} \mathrm{~N}_{3}$ & 136.08692 & -0.090 & $\mathrm{AH}$ \\
\hline & 124.98209 & 0.22 & $\mathrm{C}_{2} \mathrm{H}_{6} \mathrm{O}_{2} \mathrm{PS}$ & 124.98206 & 0.218 & $\mathrm{G}^{\prime}$ \\
\hline \multirow[t]{3}{*}{$*$} & 108.05562 & 0.04 & $\mathrm{C}_{5} \mathrm{H}_{6} \mathrm{~N}_{3}$ & 108.05562 & 0.000 & $\mathrm{AAH}$ \\
\hline & & & & Standard Deviation & 0.385 & \\
\hline & & & & Absolute Average & 0.312 & \\
\hline
\end{tabular}


Table S 15. Table showing the peak list, relative intensity, assignments, and errors (ppm) of the IRMPD fragmentation of S-metolachlor.

\begin{tabular}{|c|c|c|c|c|c|}
\hline$m / z$ & $\begin{array}{l}\text { Relative } \\
\text { Intensity }\end{array}$ & $\begin{array}{c}\text { Elemental } \\
\text { Composition }\end{array}$ & Theoretical Mass & $\begin{array}{l}\text { Error } \\
\text { (ppm) }\end{array}$ & Assignments \\
\hline 284.14121 & 22.93 & $\mathrm{C}_{15} \mathrm{H}_{23} \mathrm{ClNO}_{2}$ & 284.14118 & 0.106 & {$[\mathrm{M}+\mathrm{H}]+$} \\
\hline 252.11496 & 100.00 & $\mathrm{C}_{14} \mathrm{H}_{19} \mathrm{ClNO}$ & 252.11497 & -0.038 & A \\
\hline 224.12001 & 2.26 & $\mathrm{C}_{13} \mathrm{H}_{19} \mathrm{CIN}$ & 224.12005 & -0.182 & Rearrangement \\
\hline 212.08347 & 0.27 & $\mathrm{C}_{11} \mathrm{H}_{15} \mathrm{ClNO}$ & 212.08367 & -0.932 & D \\
\hline 176.14332 & 36.71 & $\mathrm{C}_{12} \mathrm{H}_{18} \mathrm{~N}$ & 176.14338 & -0.329 & $\mathrm{AE}$ \\
\hline 148.11202 & 0.31 & $\mathrm{C}_{10} \mathrm{H}_{14} \mathrm{~N}$ & 148.11208 & -0.354 & $\mathrm{BCE}$ \\
\hline 146.09634 & 0.21 & $\mathrm{C}_{10} \mathrm{H}_{12} \mathrm{~N}$ & 146.09643 & -0.622 & BCE \\
\hline 134.09636 & 0.59 & $\mathrm{C}_{9} \mathrm{H}_{12} \mathrm{~N}$ & 134.09643 & -0.512 & $\mathrm{DE}$ \\
\hline & & & Standard Deviation & 0.296 & \\
\hline & & & Absolute Average & 0.424 & \\
\hline
\end{tabular}


Table S 16. Table showing the peak list, relative intensity, assignments, and errors ( $\mathrm{ppm}$ ) of the EID fragmentation of S-metolachlor.

\begin{tabular}{|c|c|c|c|c|c|}
\hline $\mathbf{m} / \mathbf{z}$ & $\begin{array}{c}\text { Relative } \\
\text { Intensity }\end{array}$ & $\begin{array}{c}\text { Elemental } \\
\text { Composition }\end{array}$ & Theoretical Mass & $\begin{array}{c}\text { Error } \\
\text { (ppm) }\end{array}$ & Assignments \\
\hline 284.14591 & 100.00 & $\mathrm{C}_{15} \mathrm{H}_{23} \mathrm{ClNO}_{2}$ & 284.14118 & $16.622^{\mathrm{a}}$ & {$[\mathrm{M}+\mathrm{H}]+$} \\
\hline 252.11497 & 3.39 & $\mathrm{C}_{14} \mathrm{H}_{19} \mathrm{ClNO}$ & 252.11497 & 0.000 & $\mathrm{~A}$ \\
\hline 238.09932 & 0.33 & $\mathrm{C}_{13} \mathrm{H}_{17} \mathrm{ClNO}$ & 238.09932 & 0.000 & $\mathrm{~B}$ \\
\hline 162.12773 & 0.17 & $\mathrm{C}_{11} \mathrm{H}_{16} \mathrm{~N}$ & 162.12773 & -0.004 & $\mathrm{BE}$ \\
\hline 162.09134 & 0.60 & $\mathrm{C}_{10} \mathrm{H}_{12} \mathrm{NO}$ & 162.09134 & 0.004 & $\mathrm{DG}$ \\
\hline 160.11214 & 0.37 & $\mathrm{C}_{11} \mathrm{H}_{14} \mathrm{~N}$ & 160.11208 & 0.425 & $\mathrm{BE}$ \\
\hline & & & Standard Deviation & 0.190 & \multirow{2}{*}{} \\
\hline & & & Absolute Average & 0.087 & \multicolumn{1}{|c|}{} \\
\cline { 3 - 5 } & & & &
\end{tabular}

${ }^{a}$ The error value of this assignment is high due to the high intensity of this peak. 


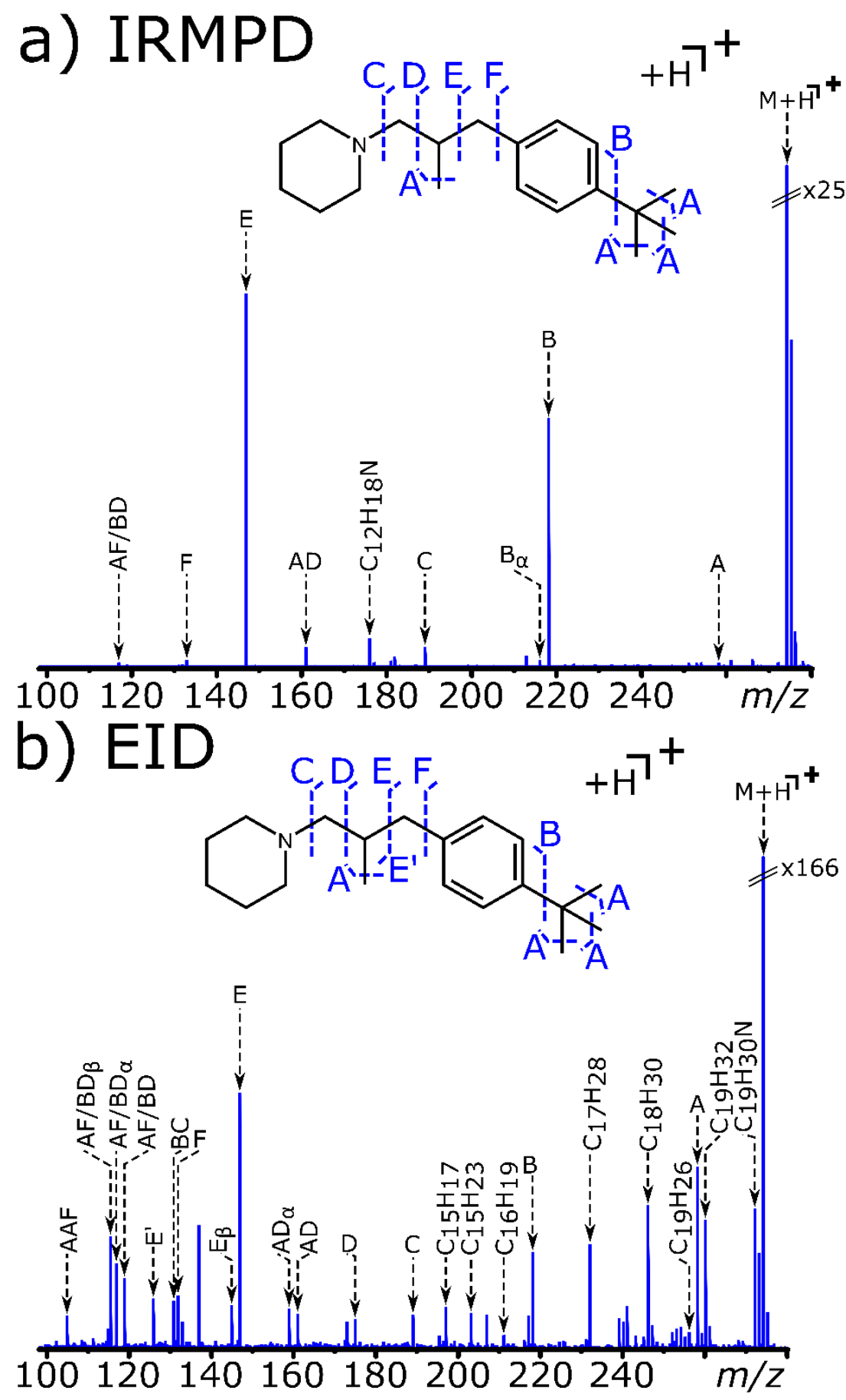

Figure S 1. Extracted fragment line spectra for fenpropidin along with their cleavage assignment diagrams for a) IRMPD and b) EID. 

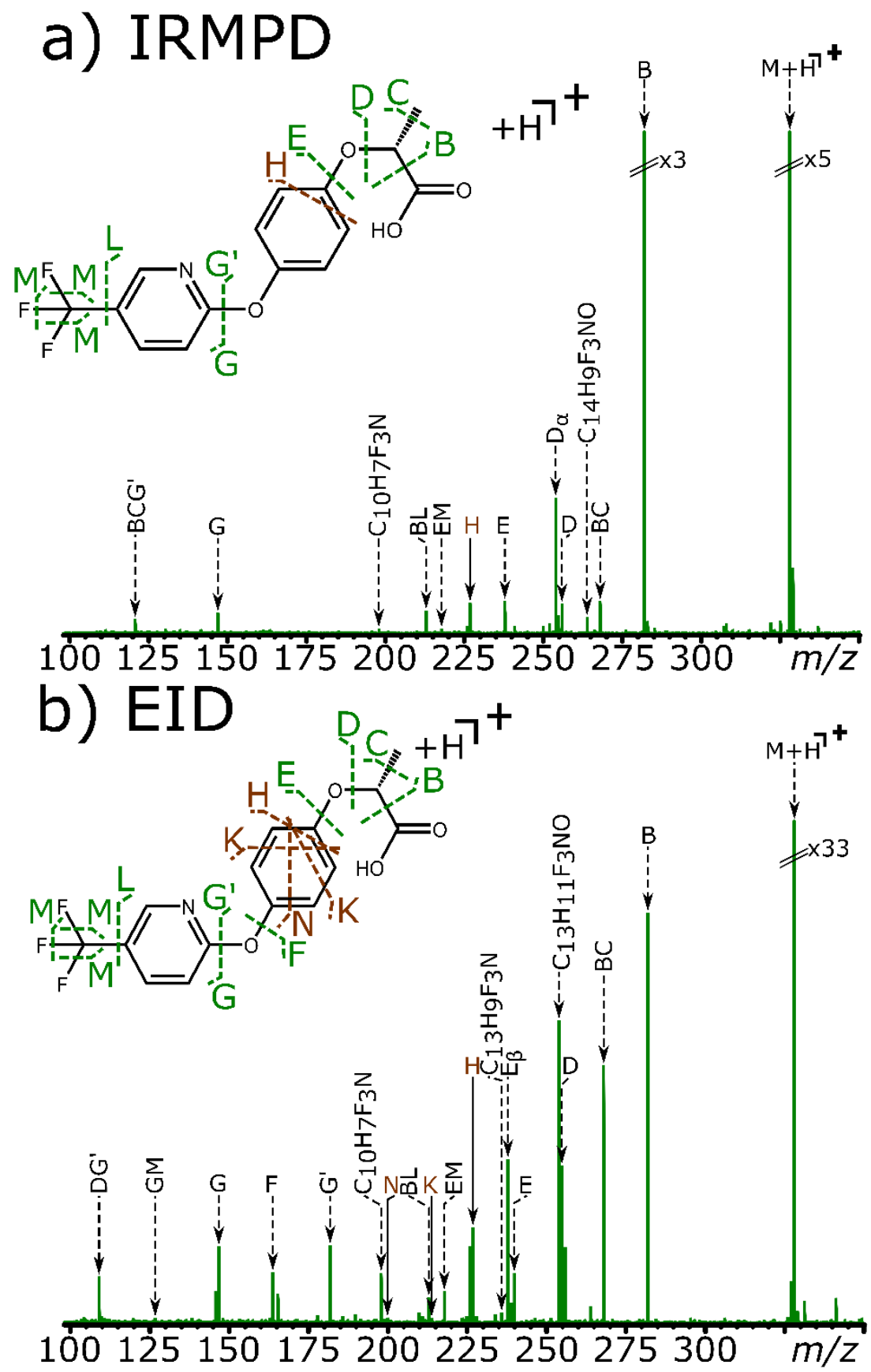

Figure S 2. Extracted fragment line spectra for fluazifop acid along with their cleavage assignment diagrams for a) IRMPD and b) EID. 
a) IRMPD
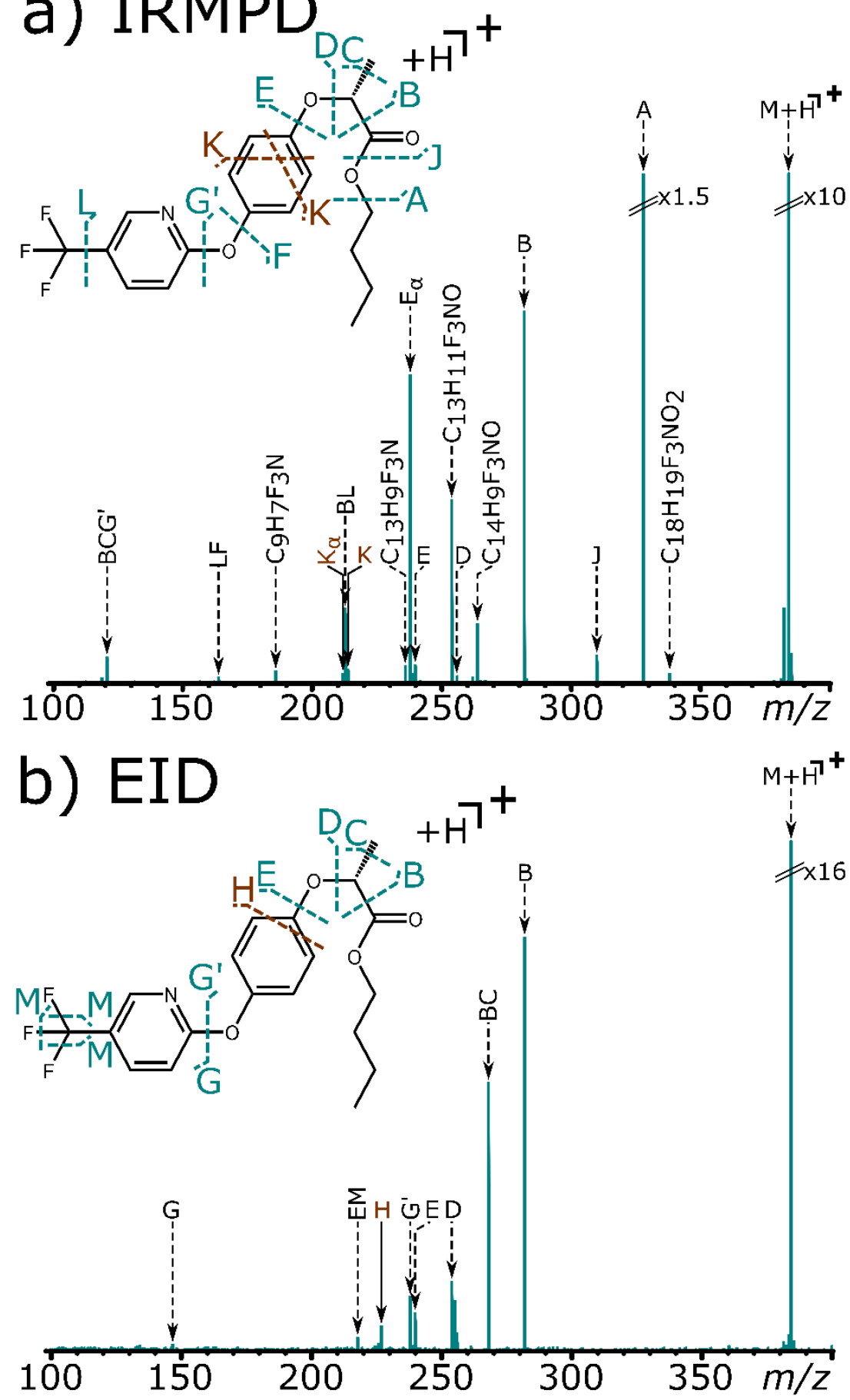

Figure S 3. Extracted fragment line spectra for fluazifop-p-butyl along with their cleavage assignment diagrams for a) IRMPD and b) EID. 


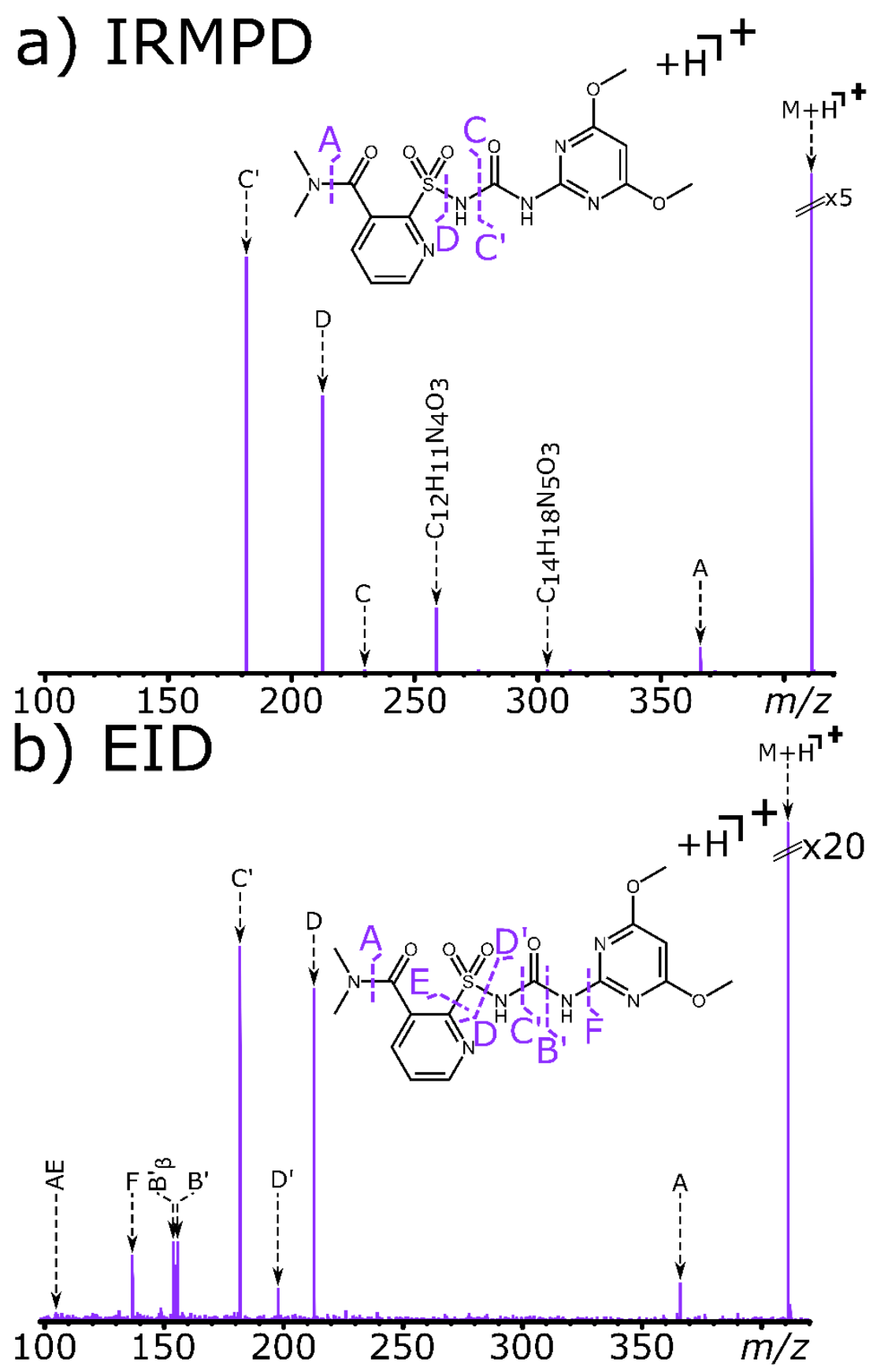

Figure S 4. Extracted fragment line spectra for nicosulfuron along with their cleavage assignment diagrams for a) IRMPD and b) EID. 


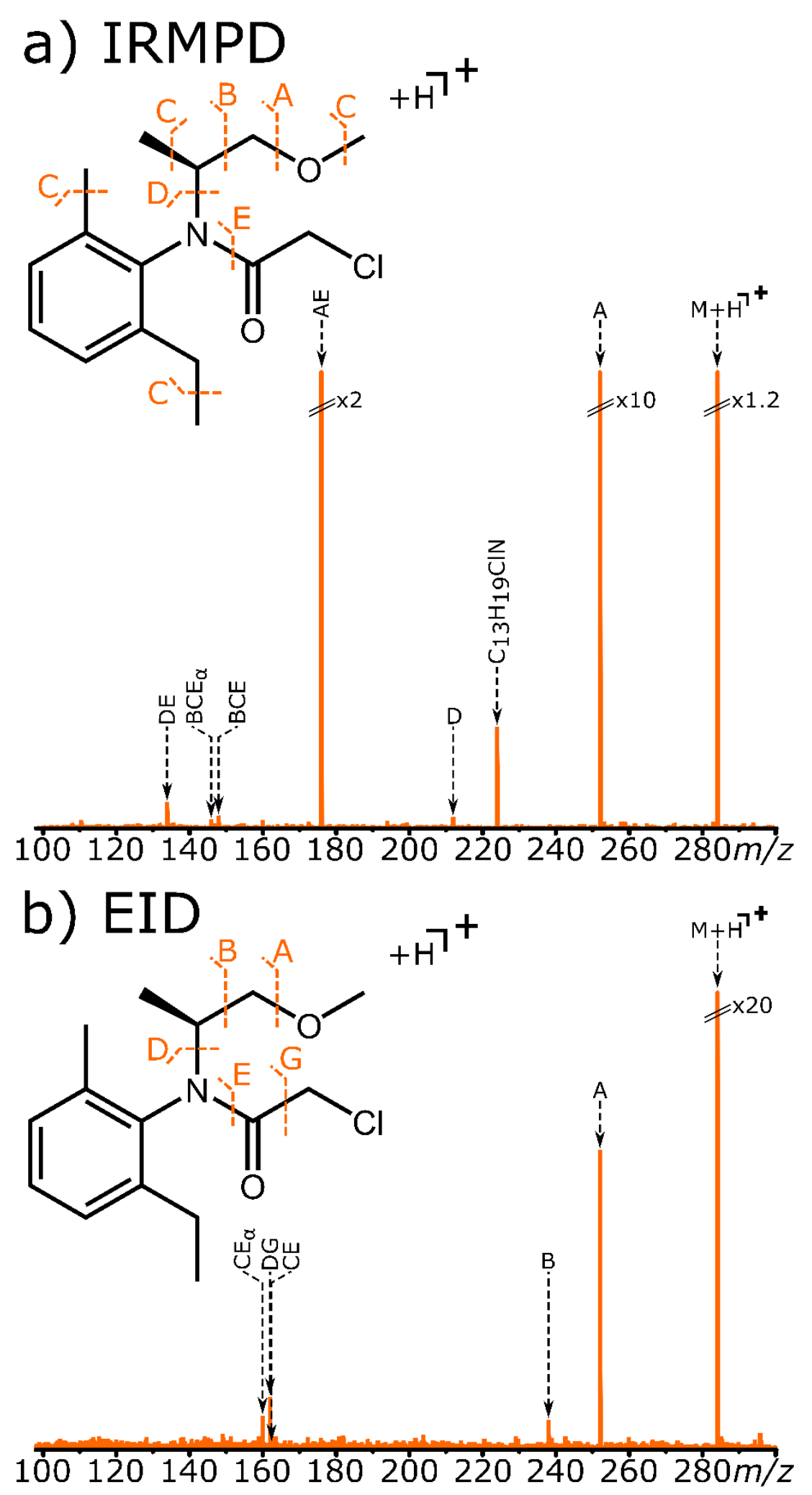

Figure S 5. Extracted fragment line spectra for S-metolachlor along with their cleavage assignment diagrams for a) IRMPD and b) EID. 


$$
F E(\%)=\left(\frac{\sum \text { All Product Ion Intensities }}{\sum \text { All Product Ions and Precursor Ion Intensities }}\right) \times 100
$$

Equation S 1: The equation used to calculate the fragmentation efficiency (FE) in percentage of the fragmentation method for each agrochemical. 